\title{
LOWER BOUNDS FOR POSITIVE ROOTS AND REGIONS OF MULTISTATIONARITY IN CHEMICAL REACTION NETWORKS
}

\author{
FRÉDÉRIC BIHAN, ALICIA DICKENSTEIN, AND MAGALÍ GIAROLI
}

\begin{abstract}
Given a real sparse polynomial system, we present a general framework to find explicit coefficients for which the system has more than one positive solution, based on the recent article by Bihan, Santos and Spaenlehauer [2]. We apply this approach to find explicit reaction rate constants and total conservation constants in biochemical reaction networks for which the associated dynamical system is multistationary.
\end{abstract}

\section{INTRODUCTION}

Multistationarity is a key property of biochemical reaction networks, because it provides a mechanism for switching between different response states. This enables multiple outcomes for cellular-decision making in cell signaling systems. Questions about steady states in biochemical reaction networks under mass-action kinetics are fundamentally questions about nonnegative real solutions to parametrized polynomial ideals. In particular, multistationarity corresponds to the existence of more than one positive steady state with fixed conserved quantities. We refer the reader to [10] for an expansion of this point of view and further references.

In this work, we develop tools from real algebraic geometry based on the paper [2] by Bihan, Santos and Spaenlehauer, to analyze systems biology models. We present a general framework to describe multistationarity regions in parameter space, that is, to find "explicit" parameters for which multistationarity occurs. We exemplify our theoretical results in different biochemical networks of interest of arbitrary size and number of variables. For this, we need to adapt the theoretical results to make them amenable to effective computations in a variety of specific instances in the modeling of biochemical systems. Our developments are also based on the existence of explicit parametrizations of the corresponding steady state varieties, as described in Theorems 28 and 35 in [24].

We give two complementary approaches. On one side, we show how to deform a given choice of reaction rate constants and total concentration constants in order to produce multistationarity. On the other side, we describe open multistationarity regions in the space of all these constants. We derive inequalities in the reaction constants and in the total conservation constants whose validity implies the presence of multistationarity. In particular, our results are the key tools in our companion paper [17] to identify multistationarity regions for enzymatic cascades of Goldbeter-Koshland loops [18] of arbitrary number of layers, when a same phosphatase catalyzes the transfer of phosphate groups at two different layers.

1.1. Basics on chemical reaction networks and multistationarity. Given a set of $s$ chemical species, a chemical reaction network on this set of species is a finite directed graph whose vertices are labeled by complexes and whose edges $\mathcal{R}$ represent the reactions and are labeled by parameters $\kappa \in \mathbb{R}_{>0}^{|\mathcal{R}|}$, which are called reaction rate constants. Complexes determine vectors in $\mathbb{Z}_{\geq 0}^{s}$ according to the stoichiometry of the species they consist of. We identify a complex with its corresponding vector and also with the formal linear combination of species specified by its coordinates. Under mass-action kinetics, the chemical reaction network defines the following autonomous system of ordinary differential equations in the concentrations $x_{1}, x_{2}, \ldots, x_{s}$ of the species as functions of time $t$ : 


$$
\dot{x}=\left(\frac{d x_{1}}{d t}, \frac{d x_{2}}{d t}, \ldots, \frac{d x_{s}}{d t}\right)=f(x)=\sum_{y \rightarrow y^{\prime} \in \mathcal{R}} \kappa_{y y^{\prime}} x^{y}\left(y^{\prime}-y\right),
$$

where $x=\left(x_{1}, x_{2}, \ldots, x_{s}\right), x^{y}=x_{1}^{y_{1}} x_{2}^{y_{2}} \ldots x_{s}^{y_{s}}$ and $y \rightarrow y^{\prime}$ indicates that $\left(y, y^{\prime}\right) \in \mathcal{R}$; that is, the complex $y$ reacts to the complex $y^{\prime}$. The right-hand side of each differential equation $\frac{d x_{i}}{d t}$ is a polynomial $f_{i}$ in $x_{1}, x_{2}, \ldots, x_{s}$ with real coefficients. A concentration vector $\bar{x} \in \mathbb{R}_{\geq 0}^{s}$ is a steady state of the system if $f(\bar{x})=0$, and $\bar{x}$ is a positive steady state if moreover $\bar{x} \in \mathbb{R}_{>0}^{s}$. We observe that the vector $\dot{x}(t)$ lies for all time $t$ in the so called stoichiometric subspace $S$ which is the linear subspace spanned by the reaction vectors $\left\{y^{\prime}-y: y \rightarrow y^{\prime} \in \mathcal{R}\right\}$. Thus, a trajectory $x(t)$ beginning at a positive vector $x(0)=x^{0} \in \mathbb{R}_{>0}^{s}$ remains in the stoichiometric compatibility class $\left(x^{0}+S\right) \cap \mathbb{R}_{\geqslant 0}^{s}$ for all $t \geq 0$. The linear equations of $x^{0}+S$ are called the conservation laws. Given a linear function $\ell$ vanishing on $S$ and any fixed stoichiometric compatibility class, $\ell$ takes a constant value over all points in this class. We will refer to these constant values as total conservation constants.

We say that the network has the capacity for multistationarity if there exists a choice of reaction rate constants $\kappa$ such that there are two or more steady states in one stoichiometric compatibility class for some initial state $x^{0}$, that is, for an appropriate choice of total conservation constants. Starting with $[7,8]$, several articles studied the capacity for multistationarity from the structure of the digraph $[1,11,12,13,20,23,24]$. Once the capacity for multistationarity is determined, the following difficult question is to find values of multistationary parameters as exhaustively and explicitly as possible. This problem is in principle effectively computable but the inherent high complexity does not allow to treat interesting networks with standard general tools. Several articles addressed this task, providing different answers based on ad-hoc computations, injectivity results based on signs of minors, and degree theory [3, 4, 5, 14, 19, 21, 28].

1.2. Our results for a two-component system. We showcase our results in a simple meaningful example. The following chemical reaction network is a two-component system [25] with hybrid histidine kinase (hybrid $H K$ ) whose multistationarity was studied in $[3,21]$. Two-component signal transduction systems enable bacteria to sense, respond, and adapt to a wide range of environments, stressors, and growth conditions. This network has six species $X_{1}, \ldots, X_{6}$, ten complexes (e.g. $X_{1}$ or $X_{1}+X_{6}$, also identified with the vectors $e_{1}$ and $e_{1}+e_{6}$ in $\mathbb{Z}_{\geq 0}^{6}$ ) and six reactions (directed edges), with labels given by positive reaction rate constants $k_{1}, \ldots, k_{6}$ :

$$
\begin{gathered}
X_{1} \stackrel{k_{1}}{\rightarrow} X_{2} \stackrel{k_{2}}{\longrightarrow} X_{3} \stackrel{k_{3}}{\longrightarrow} X_{4} \\
X_{3}+X_{5} \stackrel{k_{4}}{\longrightarrow} X_{1}+X_{6} \\
X_{4}+X_{5} \stackrel{k_{5}}{\longrightarrow} X_{2}+X_{6} \\
X_{6} \stackrel{k_{6}}{\longrightarrow} X_{5}
\end{gathered}
$$

This labeled digraph represents the following biological mechanism. Two component signaling relies on phosphotransfer reactions between histidine and aspartate residues on histidine kinases $(H K s)$ and response regulator $(R R)$ proteins. The hybrid $H K$ consists of two phosphorylable domains. We denote the phosphorylation state of each site by $p$ if the site is phosphorylated and 0 if it is not; the four possible states of $H K$ are denoted by $H K_{00}, H K_{p 0}, H K_{0 p}$, and $H K_{p p}$. We let $R R$ be the unphosphorylated response regulator protein, and $R R_{p}$ the phosphorylated form. Upon receiving a signal, the $H K$ can auto-phosphorylate. Whenever the second phosphorylation site is occupied, the phosphate group can be transferred to $R R$. In (1.2), we displayed the corresponding network of reactions denoting by $X_{1}, \ldots, X_{6}$ the chemical species $H K_{00}, H K_{p 0}, H K_{0 p}, H K_{p p}, R R, R R_{p}$, respectively. 
In what follows, we denote the concentration of the chemical species $X_{1}, \ldots, X_{6}$ by lower-case letters $x_{1}, \ldots, x_{6}$. These concentrations are assumed to be functions which evolve in time $t$, according to the following polynomial autonomous dynamical system:

$$
\begin{array}{lll}
\frac{d x_{1}}{d t}=f_{1}(x)=-k_{1} x_{1}+k_{4} x_{3} x_{5}, & \frac{d x_{2}}{d t}=f_{2}(x)=k_{1} x_{1}-k_{2} x_{2}+k_{5} x_{4} x_{5}, \\
\frac{d x_{3}}{d t}=f_{3}(x)=k_{2} x_{2}-k_{3} x_{3}-k_{4} x_{3} x_{5}, & \frac{d x_{4}}{d t}=f_{4}(x)=k_{3} x_{3}-k_{5} x_{4} x_{5}, \\
\frac{d x_{5}}{d t}=f_{5}(x)=-k_{4} x_{3} x_{5}-k_{5} x_{4} x_{5}+k_{6} x_{6}, & \frac{d x_{6}}{d t}=f_{6}(x)=k_{4} x_{3} x_{5}+k_{5} x_{4} x_{5}-k_{6} x_{6} .
\end{array}
$$

It is straightforward to check that there are two linearly independent relations: $f_{1}+f_{2}+$ $f_{3}+f_{4}=f_{5}+f_{6}=0$, which imply the existence of two constants $T_{1}, T_{2}$ such that for any value of $t$ :

$$
\begin{array}{r}
\ell_{1}(x)=x_{1}+x_{2}+x_{3}+x_{4}=T_{1}, \\
\ell_{2}(x)=x_{5}+x_{6}=T_{2} .
\end{array}
$$

We assume that the linear variety cut out by these equations intersects the positive orthant, so $T_{1}, T_{2}$ are also positive parameters. These parameters $T_{1}, T_{2}$ are the total conservation constants and the linear equations $\ell_{1}$ and $\ell_{2}$ are the conservation laws.

We now explain our strategy in the previous network (1.2). Our problem is to determine values of $\left(k_{1}, \ldots, k_{6}, T_{1}, T_{2}\right)$ in $\mathbb{R}_{>0}^{8}$ for which the polynomial system

$$
f_{1}(x)=\cdots=f_{6}(x)=\ell_{1}(x)-T_{1}=\ell_{2}(x)-T_{2}=0,
$$

has more than one positive solution $x \in \mathbb{R}_{>0}^{6}$. We have, using the framework of the main Theorems 2.9 and 2.11:

Theorem 1.1. With the notation of (1.2) and (1.3), assume that a fixed choice of reaction rate constants satisfies the condition $k_{3}>k_{1}$. Then, $k_{6}\left(\frac{1}{k_{2}}+\frac{1}{k_{3}}\right)<k_{6}\left(\frac{1}{k_{1}}+\frac{1}{k_{2}}\right)$ and for any choice of total concentration constants veriying the inequalities

$$
k_{6}\left(\frac{1}{k_{2}}+\frac{1}{k_{3}}\right)<\frac{T_{1}}{T_{2}}<k_{6}\left(\frac{1}{k_{1}}+\frac{1}{k_{2}}\right),
$$

there exist positive constants $N_{1}, N_{2}$ such that for any values of $\beta_{4}$ and $\beta_{5}$ satisfying $\beta_{4}>N_{1}$ and $\frac{\beta_{5}}{\beta_{4}}>N_{2}$, the system has at least three positive steady states after modifying only the parameters $k_{4}, k_{5}$ via the rescaling $\overline{k_{4}}=\beta_{4} k_{4}, \overline{k_{5}}=\beta_{5} k_{5}$.

In [3], the authors present necessary and sufficient conditions for the multistationarity of the network. They prove that the region of the reaction rate constant space for which multistationarity exists is completely characterized by the inequality $k_{3}>k_{1}$, but they do not describe the particular stoichiometric compatibility classes for which there are multistationarity. Instead, our inequalities (1.4) give conditions on the total concentrations and the reaction rate constants for the ocurrence of multistationarity. In [21] necessary and sufficient conditions on all the parameters of the system for bistability are provided, with a treatment ad hoc using Sturm's Theorem.

1.3. The contents of the paper. The basic idea we develop in this paper is to detect in the convex hull of the support of the monomials that define the equations of the steady states, (at least two) simplices positively decorated (see Definition 2.8) that form part of a regular subdivision. This ensures the extension of the positive real solutions of the corresponding subsystems to the total system. In Sections 2 and 3 we state and explain the theoretical setting which is of general interest for the search of positive solutions of sparse real polynomial systems beyond the applications we consider. In Section 2 we work with the same support for all the polynomials of the system. In Section 3 we present a mixed approach to the results of Section 2, considering different supports for each polynomial. 
We refer the reader to $[9,16]$ for the definitions and main properties of the combinatorial objects we deal with.

Our main results in these sections are Theorems 2.11 and 3.3. In the following sections, we apply these results for a class of biochemical reaction networks under mass-action kinetics. This application is not straightforward and requires known and new results on the structure of their steady states.

In Section 4, we study the sequential distributive multisite phosphorylation systems with any number $n$ of phosphorylation sites. Such systems were studied by many authors, starting with Wang and Sontag [28], who gave bounds and conditions for monostationarity and multistationarity in the parameters, with an interesting treatment ad hoc which also allowed them to find improved lower bounds (see also [19]). In [5], Conradi and Mincheva showed using degree theory and computations with the aid of a computer algebra system, that catalytic constants determine the capacity for multistationarity in the dual phosphorylation mechanism. They also indicate in the case $n=2$ how to find values of the total concentrations such that multistationarity occurs. The more general interesting approach in [3] is also based on degree theory. The authors show how to find conditions on the reaction rates to guarantee mono or multistationarity, but they do not describe the particular total concentration constants for which there are multiple equilibria. With our approach, we obtain for any $n$ a system of three polynomial equations in three variables that describes the steady states, in the framework of [2]. We give conditions on all the parameters (both on the reaction constants and on the total concentration constants) so that there are at least two positively decorated simplices in a regular subdivision of the convex hull of our support and by rescaling the rest of the parameters, we guarantee the existence of at least two non-degenerate positive steady states (see Theorem 4.1).

Then, in Section 5, we show that these systems as well as the two-component system in the Introduction, are a particular example of a class of biological systems introduced in [24] called MESSI systems, which contains many other important mechanisms. We focus on a particular class of MESSI systems called $s$-toric MESSI systems, which includes the sequential phosphorylation systems, for which explicit monomial parametrizations of the steady states are given in [24]. We prove general results for $s$-toric MESSI systems, that in particular explain our computations in Section 4. Theorem 5.4 is the key to apply the framework of Theorem 2.11 to describe multistationarity regions for all these biological systems.

\section{Positive SOlutions of SPARSE POlynomial SYstems}

Along this section, we fix a finite point configuration

$$
\mathcal{A}=\left\{a_{1}, \ldots, a_{n}\right\} \subset \mathbb{Z}^{d}, n \geq d+2,
$$

and we assume that the convex hull of $\mathcal{A}$ is a full-dimensional polytope. A subset of $\mathcal{A}$ consisting of affinely independent points will be called a simplex; we will also say that it is a $d$-simplex when the dimension of its convex hull is $d$.

2.1. Regular subdivisions. A regular subdivision of $\mathcal{A}$ is induced by a height function $h: \mathcal{A} \rightarrow \mathbb{R}, h=\left(h\left(a_{1}\right), \ldots, h\left(a_{n}\right)\right)$ as follows. Consider the lower convex hull of the lifted configuration $\mathcal{A}^{h}=\left\{\left(a_{1}, h\left(a_{1}\right)\right), \ldots,\left(a_{n}, h\left(a_{n}\right)\right)\right\} \subset \mathbb{R}^{d+1}$, which is the union of the faces of the convex hull of $\mathcal{A}^{h}$ for which the inner normal directions have positive last coordinate. The associated regular subdivision $\Gamma_{h}$ is the union of the subsets $\mathcal{A}_{F}=\left\{a_{i}:\left(a_{i}, h\left(a_{i}\right)\right) \in\right.$ $F\}$ of $\mathcal{A}$ which are the projections back to $\mathcal{A}$ of lifted points in a face $F$ of this lower convex hull.

It is useful to have a more geometric picture of this subdivision, like the one depicted in Figure 1, but it is important to note that these subsets cannot be identified in general with their convex hulls, which are convex polytopes with integer vertices, but with their marked convex hulls containing all the points $a_{j} \in \mathcal{A}$ for which the affine linear function which interpolates the values of $h$ at the vertices takes the value $h\left(a_{j}\right)$ at $a_{j}$ (so, other points 

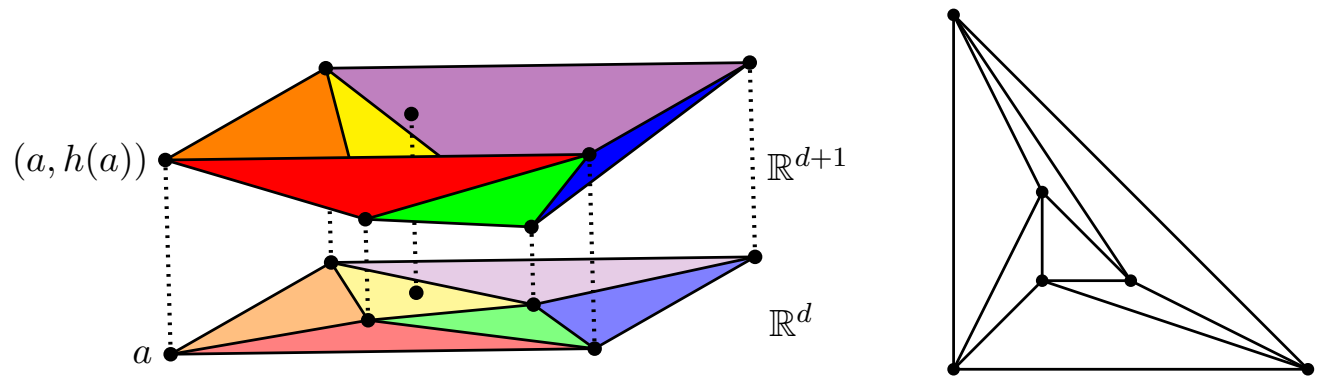

FiguRe 1. Regular triangulation.

FigURE 2. Non-regular triangulation.

besides the vertices can occur). A regular subdivision is called a regular triangulation of $\mathcal{A}$ if the only points in each subset of the subdivision are the vertices of their convex hull and these vertices are affinely independent. Figure 2 depicts a triangulation into simplices which is not regular, that is, which cannot be induced by any height function $h$.

The set of all height vectors inducing a regular subdivision $\Gamma$ of $\mathcal{A}$ is defined by a finite number of linear inequalities. Thus, this set is a finitely generated convex cone $\mathcal{C}_{\Gamma}$ in $\mathbb{R}^{n}$ with apex at the origin. When $\Gamma$ is a triangulation, the cone $\mathcal{C}_{\Gamma}$ is full dimensional (cut out by strict inequalities). All these facts and many more are described in [16, Ch.7].

We will denote by $A \in \mathbb{Z}^{(d+1) \times n}$ the integer matrix:

$$
A=\left(\begin{array}{ccc}
1 & \ldots & 1 \\
a_{1} & \ldots & a_{n}
\end{array}\right)
$$

and by $A_{h} \in \mathbb{R}^{(d+2) \times n}$ the matrix:

$$
A_{h}=\left(\begin{array}{ccc}
1 & \ldots & 1 \\
a_{1} & \ldots & a_{n} \\
h_{1} & \ldots & h_{n}
\end{array}\right) .
$$

Note that our assumption that the convex hull of $\mathcal{A}$ has dimension $d$ is equivalent to assuming that the rank of $A$ is equal to $d+1$.

Let $\Delta=\left\{a_{i_{1}}, \ldots, a_{i_{d+1}}\right\}$ be a $d$-simplex with vertices in $\mathcal{A}$. Let $I=\left\{i_{1}, i_{2}, \ldots, i_{d+1}\right\}$ and assume $i_{1}<i_{2}<\cdots<i_{d+1}$. Denote by $d_{I}$ the determinant of the $(d+1) \times(d+1)$ submatrix of $A$ with columns indicated by $I$, which is nonzero because we are assuming that $\Delta$ is a simplex. Also, for any index $i \notin I$, denote by $d_{I \cup\{i\}}(h)$ the determinant of the $(d+2) \times(d+2)$ submatrix of $A_{h}$ with columns indicated by $I \cup\{i\}$, multiplied by the sign of the permutation that sends the set of indices in $I \cup\{i\}$ ordered by $<$ to $\left(i_{1}, \ldots, i_{d+1}, i\right)$ with $i$ as the last index. Using the Laplace expansion of the determinant along the last row, we see that $d_{I \cup\{i\}}(h)$ is an affine linear function of $h$ which equals $\left\langle m_{i}^{I}, h\right\rangle$, where $m_{i}^{I}$ is a vector in the kernel of $A$ with support included $I \cup\{i\}$ and with nonzero $i$-th coordinate, for any $i \notin I$.

Consider the cone $\mathcal{C}_{\Delta}$ of all height vectors inducing a regular subdivision of $\mathcal{A}$ that contains $\Delta$. Observe that $\mathcal{C}_{\Delta}$ is non-empty; for instance, any vector $h \in \mathbb{R}^{n}$ with $h_{i}=0$ for any $i \in I$ and $h_{i}>0$ for any index $i \notin I$, belongs to $\mathcal{C}_{\Delta}$. Moreover, $C_{\Delta}$ is an open rational polyhedral cone, described as follows:

Lemma 2.1. With the previous notations, we have:

$$
\mathcal{C}_{\Delta}=\left\{\left(h_{1}, \ldots, h_{n}\right) \in \mathbb{R}^{n}:\left\langle d_{I} \cdot m_{i}^{I}, h\right\rangle=d_{I} \cdot d_{I \cup\{i\}}(h)>0\right\},
$$

and the $n-(d+1)$ vectors $d_{I} \cdot m_{i}^{I}, i \notin I$, are a basis of the kernel of $A$.

The proof of Lemma 2.1 is straightforward. Let $p \geq 1$ and consider $\Delta_{1}, \ldots, \Delta_{p} d$ simplices in $\mathcal{A}$. We denote by $\mathcal{C}_{\Delta_{1}, \ldots, \Delta_{p}}$ the cone of all height vectors $h$ defining a regular 

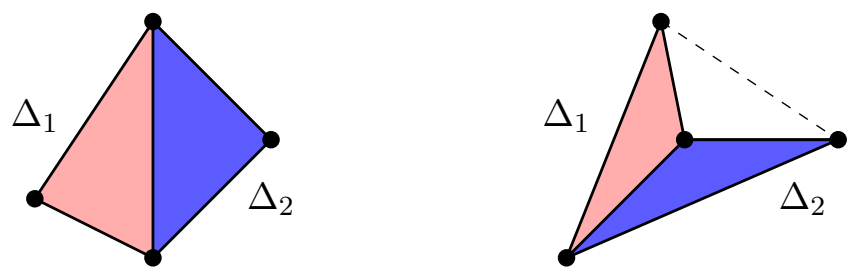

Figure 3 . Examples of simplices $\Delta_{1}$ and $\Delta_{2}$, which share a facet, $d=2$.

subdivision of $\mathcal{A}$ that contains $\Delta_{1}, \ldots, \Delta_{p}$. We deduce from Lemma 2.1 the following description.

Lemma 2.2. Let $\Delta_{1}, \ldots, \Delta_{p}$ be simplices in $\mathcal{A}$ which occur in a regular subdivision of $A$. If the index set of the vertices of $\Delta_{k}$ is $I_{k}$ for any $k=1, \ldots, p$, then the nonempty open polyhedral cone $\mathcal{C}_{\Delta_{1}, \ldots, \Delta_{p}}$ is defined by the linear inequalities

$$
\left\langle d_{I_{k}} \cdot m_{i}^{I_{k}}, h\right\rangle>0 \quad \text { for all } k=1, \ldots, p \text {, and all } i \notin I_{k},
$$

and the vectors $\left\{d_{I_{k}} \cdot m_{i}^{I_{k}}, k=1, \ldots, p, i \notin I_{k}\right\}$ generate the kernel of $A$.

Definition 2.3. We will say that two simplices $\Delta_{1}, \Delta_{2} \subset \mathcal{A}$ share a facet if the intersection of their convex hulls is a facet of both, that is, a face of codimension one. See Figure 3.

We will need the following remark:

Remark 2.4. A point configuration $\mathcal{B}=\left\{b_{1}, \ldots, b_{d+2}\right\}$ with $d+2$ points which span $\mathbb{R}^{d}$ and such that any proper subset is affinely independent, is called a circuit. Any circuit $\mathcal{B}$ has exactly two triangulations $\Gamma_{ \pm}$, which are furthermore regular. They can be described in the following way (see [16, chapter 7, Proposition 1.2.]). Consider any nonzero vector $\lambda \in \mathbb{R}^{d+2}$ such that $\sum_{i=1}^{d+2} \lambda_{i}=0$ and $\sum_{i=1}^{d+2} \lambda_{i} b_{i}=0$ (in other words, any nontrivial affine relation on $\mathcal{B})$. Note that all coordinates of $\lambda$ are nonzero. Write $[d+2]=\{1, \ldots, d+2\}$ as the disjoint union $N_{+} \sqcup N_{-}$, with $N_{+}=\left\{i \in[d+2]: \lambda_{i}>0\right\}$ and $N_{-}=\left\{i \in[d+2]: \lambda_{i}<0\right\}$. The $d$-simplices of $\Gamma_{+}$are the sets $[d+2] \backslash\{i\}$ for $i \in N_{+}$. Similarly, the $d$-simplices of $\Gamma_{-}$are the sets $[d+2] \backslash\{i\}$ for $i \in N_{-}$.

We are ready to prove the following proposition, that we will need in our applications.

Proposition 2.5. Let $\Delta_{1}, \Delta_{2}$ be two d-simplices in $\mathcal{A}$ which share a facet. Then, there exists a regular subdivision of $\mathcal{A}$ containing $\Delta_{1}$ and $\Delta_{2}$, and so the cone $\mathcal{C}_{\Delta_{1}, \Delta_{2}}$ is nonempty. Moreover, there exists a regular triangulation containg both simplices.

Proof. The configuration $\mathcal{B}=\Delta_{1} \cup \Delta_{2}$ has cardinality $d+2$ and it is a circuit. As we remarked $\mathcal{B}$ has exactly two regular triangulations $\Gamma_{ \pm}$. Without loss of generality, assume $\mathcal{B}=\left\{a_{1}, \ldots, a_{d+2}\right\}$, with $F=\left\{a_{1}, \ldots, a_{d}\right\}$ the common facet of $\Delta_{1}$ and $\Delta_{2}$. Let $\lambda \in \mathbb{Z}^{d+2}$ be a nontrivial affine relation on $\mathcal{B}$. As $a_{d+1}$ and $a_{d+2}$ lie in opposite sides of the hyperplane passing through $F$, it holds that $\lambda_{d+2}$ and $\lambda_{d+1}$ have the same sign. Therefore $\Delta_{1}$ and $\Delta_{2}$ belong to the same regular triangulation, say $\Gamma_{+}$.

Let $h: \mathcal{B} \rightarrow \mathbb{R}$ be a height function inducing $\Gamma_{+}$. Let $\varphi_{1}, \ldots, \varphi_{\ell}$ be the affine linear functions which interpolate the values of $h$ at each of the $d$-simplices of $\Gamma_{+}$and set $\varphi=$ $\max \left\{\varphi_{1}, \ldots, \varphi_{\ell}\right\}$. For any choice of generic positive values $h_{d+3}, \ldots, h_{n}$ verifying $h_{j}>$ $\varphi\left(a_{j}\right)$ for any $j=d+3, \ldots, n$, the height function $h^{\prime}: \mathcal{A} \rightarrow \mathbb{R}$ that extends $h$ by defining $h^{\prime}\left(a_{j}\right)=h_{j}, j=d+3, \ldots, n$, induces a regular triangulation of $\mathcal{A}$ extending $\Gamma_{+}$, and so in particular, it contains $\Delta_{1}$ and $\Delta_{2}$.

Remark 2.6. Under the notations of Lemma 2.2 with $p=2$, if $\Delta_{1}$ and $\Delta_{2}$ share a common facet with vertices $a_{i}$ with $i \in I$, then the inequality corresponding to $k=1$ and $i \in I_{2} \backslash I$ coincides with the one corresponding to $k=2$ and $i \in I_{1} \backslash I$, so that we might forget one of these inequalities in (2.3) to get $2(n-d-1)-1=2 n-2 d-3$ inequalities defining $\mathcal{C}_{\Delta_{1}, \Delta_{2}}$. This generalizes the circuit case where any of the two regular triangulations is determined by one of its simplices. 
2.2. Decorated simplices and lower bounds for the number of positive solutions. Consider a sparse polynomial system in $d$ variables $x=\left(x_{1}, \ldots, x_{d}\right)$ with support included in $\mathcal{A}$ and coefficient matrix $C=\left(c_{i j}\right) \in \mathbb{R}^{d \times n}$ :

$$
f_{1}(x)=\cdots=f_{d}(x)=0,
$$

with

$$
f_{i}(x)=\sum_{j=1}^{n} c_{i j} x^{a_{j}} \in \mathbb{R}\left[x_{1}, \ldots, x_{d}\right], i=1, \ldots, d .
$$

Let $\Gamma$ be a regular subdivision of $\mathcal{A}$ and $h \in \mathcal{C}_{\Gamma}$. Consider the following family of polynomial systems parametrized by a positive real number $t$ :

$$
f_{1, t}(x)=\cdots=f_{d, t}(x)=0,
$$

where

$$
f_{i, t}(x)=\sum_{j=1}^{n} c_{i j} t^{h\left(a_{j}\right)} x^{a_{j}} \in \mathbb{R}\left[x_{1}, \ldots, x_{d}\right], i=1, \ldots, d, t>0 .
$$

For each positive real value of $t$, this system has again support included in $\mathcal{A}$. Recall that a common root of (2.5) is nondegenerate when it is not a zero of the Jacobian of $f_{1, t}, \ldots, f_{d, t}$.

Following Section 3 in [2], we define:

Definition 2.7. $A d \times(d+1)$ matrix $M$ with real entries is called positively spanning if all the values $(-1)^{i} \operatorname{minor}(M, i)$ are nonzero and have the same sign, where $\operatorname{minor}(M, i)$ is the determinant of the square matrix obtained by removing the $i$-th column.

Equivalently, a matrix is positively spanning if all the coordinates of any non-zero vector in the kernel of the matrix are non-zero and have the same sign.

Definition 2.8. Let $C$ be a $d \times n$ matrix with real entries. We say that a d-simplex $\Delta=\left\{a_{i_{1}}, \ldots, a_{i_{d+1}}\right\}$ in $\Gamma$ is positively decorated by $C$ if the $d \times(d+1)$ submatrix of $C$ with columns indicated by $\left\{i_{1}, \ldots, i_{d+1}\right\}$ is positively spanning.

The following result is a generalization of Theorem 3.4 in [2], with essentially the same proof, combined with Proposition 2.5:

Theorem 2.9. Let $\mathcal{A}$ and $p$ d-simplices $\Delta_{1}, \ldots, \Delta_{p}$ which occur in a regular subdivision $\Gamma$ of $\mathcal{A}$, and which are positively decorated by a matrix $C \in \mathbb{R}^{d \times n}$. Let $h$ be a height function that defines $\Gamma$. Then, there exists $t_{0} \in \mathbb{R}_{>0}$ such that for all $0<t<t_{0}$, the number of (nondegenerate) solutions of (2.5) contained in the positive orthant is at least $p$. In particular, if there are two d-simplices with vertices in $\mathcal{A}$ sharing a facet which are both positively decorated by $C$, the number of positive solutions of (2.5) is at least two.

The idea of the proof consists first in observing that the system obtained by considering only the monomials in a positively decorated $d$-simplex has exactly one nondegenerate positive solution. Then, we can take a compact set $K$ in the positive orthant which contains all the nondegenerate positive solutions of the restricted systems, for each positively decorated $d$-simplex. If $t>0$ is small enough, we can obtain pairwise disjoint sets (of the form $t^{\alpha} \dot{K}, \alpha \in \mathbb{R}^{d}$ ), such that each one contains at least one non-degenerate positive of the system (2.5). We note that in [2], the result is for a regular triangulation but it also holds if we have a regular subdivision.

We will give a similar result in Theorem 2.11 below, but our focus is to describe a subset with nonempty interior in the space of coefficients where we can bound from below the number of positive solutions of the associated system. We start with a general result about convex polyhedral cones.

Proposition 2.10. Let $L$ be a linear subspace of $\mathbb{R}^{n}$ of dimension $\ell_{1}$ together with a basis $\left\{v_{1}, \ldots, v_{\ell_{1}}\right\}$. Let $m_{1}, \ldots, m_{\ell}$ be a system of generators of $L^{\perp}$ such that the open polyhedral cone

$$
\mathcal{C}=\left\{h \in \mathbb{R}^{n}:\left\langle m_{r}, h\right\rangle>0, \quad r=1, \ldots, \ell\right\}
$$


is non-empty. For any $\varepsilon \in \mathbb{R}_{>0}^{\ell}$, denote by $\mathcal{C}_{\varepsilon}$ the $n$-dimensional convex polyhedral cone

$$
\mathcal{C}_{\varepsilon}=\left\{h \in \mathbb{R}^{n}:\left\langle m_{r}, h\right\rangle>\varepsilon_{r}, \quad r=1, \ldots, \ell\right\} .
$$

Consider the map $\varphi: \mathbb{R}_{>0}^{\ell_{1}} \times \mathbb{R}_{>0} \times \mathbb{R}^{n} \rightarrow \mathbb{R}_{>0}^{n}$ :

$$
\varphi(\alpha, t, h)=\left(t^{h_{1}} \prod_{j=1}^{\ell_{1}} \alpha_{j}^{v_{j 1}}, \ldots, t^{h_{n}} \prod_{j=1}^{\ell_{1}} \alpha_{j}^{v_{j n}}\right) .
$$

Then, we have:

$$
\begin{aligned}
& \varphi\left(\mathbb{R}_{>0}^{\ell_{1}} \times\left(0, t_{0}\right) \times \mathcal{C}_{\varepsilon}\right)=\left\{\gamma \in \mathbb{R}_{>0}^{n}: \gamma^{m_{r}}<t_{0}^{\varepsilon_{r}}, r=1 \ldots, \ell\right\} \text { and } \\
& \varphi\left(\mathbb{R}_{>0}^{\ell_{1}} \times\left(0, t_{0}\right] \times \overline{\mathcal{C}}_{\varepsilon}\right)=\left\{\gamma \in \mathbb{R}_{>0}^{n}: \gamma^{m_{r}} \leq t_{0}^{\varepsilon_{r}}, r=1 \ldots, \ell\right\},
\end{aligned}
$$

where $\overline{\mathcal{C}}_{\varepsilon}$ denotes the closure of $\mathcal{C}_{\varepsilon}$.

Proof. We first prove that a positive vector $\gamma$ is of the form $\gamma=\varphi(\alpha, t, h)$ if and only if

$$
\gamma^{m_{r}}=t^{\left\langle m_{r}, h\right\rangle}, \quad r=1, \ldots, \ell
$$

The only if part is straightforward, taking into account that we are assuming that for any $r, j$ it holds that $\left\langle m_{r}, v_{j}\right\rangle=0$ :

$$
\varphi(\alpha, t, h)^{m_{r}}=t^{\left\langle m_{r}, h\right\rangle} \prod_{j=1}^{\ell_{1}} \alpha_{j}^{\left\langle m_{r}, v_{j}\right\rangle}=t^{\left\langle m_{r}, h\right\rangle} .
$$

On the other side, if $\gamma^{m_{r}}=t^{\left\langle m_{r}, h\right\rangle}$ for any $r=1, \ldots, \ell$, then the vector

$$
\gamma_{t, h}=\left(\gamma_{1} t^{-h_{1}}, \ldots, \gamma_{n} t^{-h_{n}}\right)
$$

verifies that $\gamma_{t, h}^{m}=1$, for any $m \in L^{\perp}$. Thus, taking coordinatewise logarithms, we get that

$$
\left\langle m, \log \left(\gamma_{t, h}\right)\right\rangle=0 \text { for any } m \in L^{\perp},
$$

which means that $\log \left(\gamma_{t, h}\right) \in L$. Then, there exist real constants $\lambda_{1}, \ldots, \lambda_{\ell}$ such that $\log \left(\gamma_{t, h}\right)=\sum_{j=1}^{\ell} \lambda_{j} v_{j}$. Calling $\alpha \in \mathbb{R}_{>0}^{\ell}$ the vector with coordinates $\alpha_{j}=e^{\lambda_{j}}$ we get that $\gamma=\varphi(\alpha, t, h)$, as wanted.

Now, assuming $0<t<t_{0}<1$ and $\left\langle m_{r}, h\right\rangle>\varepsilon_{r}$ for all $r=1 \ldots, \ell$, we have that $t^{\left\langle m_{r}, h\right\rangle}<t_{0}^{\varepsilon_{r}}$ and moreover $\left(0, t_{0}^{\varepsilon_{r}}\right)=\left\{t^{\left\langle m_{r}, h\right\rangle}, 0<t<t_{0}, h \in \mathcal{C}_{\varepsilon}\right\}$, which proves both containments. The other equality follows immediately.

We now present the main result of this section.

Theorem 2.11. Consider a set $\mathcal{A}=\left\{a_{1}, \ldots, a_{n}\right\}$ of $n$ points in $\mathbb{R}^{d}$ and a matrix $C=$ $\left(c_{i, j}\right) \in \mathbb{R}^{d \times n}$. Assume there are $p d$-simplices $\Delta_{1}, \ldots, \Delta_{p}$ contained in $\mathcal{A}$, which are part of a regular subdivision of $\mathcal{A}$ and are positively decorated by a $d \times n$ matrix $C$.

Let $m_{1} \ldots, m_{\ell} \in \mathbb{R}^{n}$ be vectors that define a presentation of the cone $\mathcal{C}_{\Delta_{1}, \ldots, \Delta_{p}}$ of all height vectors $h \in \mathbb{R}^{n}$ that induce a regular subdivision of $\mathcal{A}$ containing $\Delta_{1}, \ldots, \Delta_{p}$ :

$$
\mathcal{C}_{\Delta_{1}, \ldots, \Delta_{p}}=\left\{h \in \mathbb{R}^{n}:\left\langle m_{r}, h\right\rangle>0, r=1, \ldots, \ell\right\} .
$$

Then, for any $\varepsilon \in(0,1)^{\ell}$ there exists $t_{0}(\varepsilon)>0$ such that for any $\gamma$ in the open set

$$
U=\cup_{\varepsilon \in(0,1)^{\ell}}\left\{\gamma \in \mathbb{R}_{>0}^{n} ; \gamma^{m_{r}}<t_{0}(\varepsilon)^{\varepsilon_{r}}, r=1 \ldots, \ell\right\},
$$

the system

$$
\sum_{j=1}^{n} c_{i j} \gamma_{j} x^{a_{j}}=0, \quad i=1, \ldots, d,
$$

has at least $p$ nondegenerate solutions in the positive orthant. In particular, given two $d$-simplices that share a facet, the system (2.10) has at least 2 nondegenerate positive solutions. 
Proof. Let $L$ be the linear subspace generated by the rows of the matrix $A$, and let $v_{1}, \ldots, v_{d+1}$ denote its row vectors, which are a basis of $L$ because we are assuming that $A$ has rank $d+1$. With this choice, we can apply Proposition 2.10 to the cone $\mathcal{C}=\mathcal{C}_{\Delta_{1}, \ldots, \Delta_{p}}$, by Lemma 2.2. Note that the map $\varphi: \mathbb{R}_{>0}^{d+1} \times \mathbb{R}_{>0} \times \mathbb{R}^{n} \rightarrow \mathbb{R}_{>0}^{n}$ equals in this case:

$$
\varphi(\alpha, t, h)=\left(\alpha^{\left(1 a_{1}\right)} t^{h_{1}}, \ldots, \alpha^{\left(1 a_{n}\right)} t^{h_{n}}\right) .
$$

We denote by $\overline{\mathcal{C}}_{\varepsilon}$ the closure of the cone $\mathcal{C}_{\varepsilon}$ defined in (2.6). Let $B$ denote the unit ball in $\mathbb{R}^{n}$. In the proof of Theorem 3.4 in [2], and thus in the proof of Theorem 2.9, one can see that given any $h$, it is possible to find a positive number $t_{0}$ for which the conclusion of Theorem 2.9 holds for any $h^{\prime}$ close to $h$. As $B \cap \overline{\mathcal{C}}_{\varepsilon}$ is compact, there exists $t_{1}(\varepsilon) \in(0,1)$ such that the conclusion holds for any $t \in\left(0, t_{1}(\varepsilon)\right)$ and any $h \in B \cap \overline{\mathcal{C}}_{\varepsilon}$. But if $h \in \overline{\mathcal{C}}_{\varepsilon}$ satisfies $\|h\|>1$, we can write it as $h=\|h\| h^{\prime}$, with $h^{\prime} \in B \cap \overline{\mathcal{C}}_{\varepsilon}$. Then, for any $t \in(0,1)$, $t^{h}=\left(t^{\|h\|}\right)^{h^{\prime}}$, with $0<t^{\|h\|}<t$ and so the conclusion of Theorem 2.9 holds for any $h \in \overline{\mathcal{C}}_{\varepsilon}$ provided $t \in\left(0, t_{1}(\varepsilon)\right]$. By Proposition 2.10, the image by $\varphi$ of $\mathbb{R}_{>0}^{d+1} \times\left(0, t_{1}(\varepsilon)\right] \times \overline{\mathcal{C}}_{\varepsilon}$ equals $\left\{\gamma \in \mathbb{R}_{>0}^{n} ; \gamma^{m_{r}} \leq t_{0}(\varepsilon)^{\varepsilon_{r}}, r=1 \ldots, \ell\right\}$. Note also that $\mathcal{C}_{\Delta_{1}, \ldots, \Delta_{p}}=\cup_{\varepsilon} \overline{\mathcal{C}}_{\varepsilon}$.

Observe that if $\gamma=\varphi(\alpha, t, h)$, then for any $j=1, \ldots, n$,

$$
\gamma_{j} x^{a_{j}}=\alpha^{\left(1 a_{j}\right)} x^{a_{j}}=\alpha_{1} t^{h_{j}} y^{a_{j}},
$$

where $y_{i}=\alpha_{i+1} x_{i}$ for any $i=1, \ldots, d$. As all $\alpha_{i}>0$, system (2.10) has the same number of positive solutions as

$$
\sum_{j=1}^{n} c_{i j} t^{h_{j}} y^{a_{j}}=0, \quad i=1, \ldots, d,
$$

which is at least $p$ for $t \in\left(0, t_{1}(\varepsilon)\right]$.

Remark 2.12. Theorem 2.11 says that if we choose $\varepsilon \in(0,1)^{\ell}$, then there exists positive real numbers $M_{1}=M_{1}(\varepsilon), \ldots, M_{r}=M_{r}(\varepsilon)$, such that system (2.10) has at least $p$ nondegenerate solutions in the positive orthant for any vector $\gamma$ in $\mathbb{R}_{>0}^{n}$ satisfying

$$
\gamma^{m_{r}}<M_{r} \text { for } r=1, \ldots, \ell \text {. }
$$

We have to remark that the choice of the positive constants $M_{1}, \ldots, M_{r}$ is not algorithmic, but our result makes clear that there is an open set in coefficient space for which many positive solutions can be found, and inequalities (2.12) indicate "in which directions" the coefficients have to be scaled in order to get at least as many positive solutions as the number of decorated simplices.

As a first application of Theorems 2.9 and 2.11, we give a proof of Theorem 1.1, corresponding to the example of the two component system with Hybrid Histidine Kinase (1.2) in the Introduction.

Proof of Theorem 1.1. From $f_{2}=f_{3}=f_{4}=f_{5}=0$ we get:

$$
x_{1}=\frac{k_{4} k_{5} x_{4} x_{5}^{2}}{k_{1} k_{3}}, x_{2}=\frac{k_{4} k_{5} x_{4} x_{5}^{2}}{k_{2} k_{3}}+\frac{k_{5} x_{4} x_{5}}{k_{2}}, x_{3}=\frac{k_{5} x_{4} x_{5}}{k_{3}}, x_{6}=\frac{k_{4} k_{5} x_{4} x_{5}^{2}}{k_{3} k_{6}}+\frac{k_{5} x_{4} x_{5}}{k_{6}} .
$$

Then, at steady state, the concentrations of the species can be obtained from the values of $x_{4}$ and $x_{5}$. If we replace these expressions into the conservation laws (1.3), we get the equations:

$$
\begin{aligned}
\frac{k_{4} k_{5} x_{4} x_{5}^{2}}{k_{1} k_{3}}+\frac{k_{4} k_{5} x_{4} x_{5}^{2}}{k_{2} k_{3}}+\frac{k_{5} x_{4} x_{5}}{k_{2}}+\frac{k_{5} x_{4} x_{5}}{k_{3}}+x_{4}-T_{1} & =0, \\
x_{5}+\frac{k_{4} k_{5} x_{4} x_{5}^{2}}{k_{3} k_{6}}+\frac{k_{5} x_{4} x_{5}}{k_{6}}-T_{2} & =0 .
\end{aligned}
$$

We can write this system in matricial form:

$$
C\left(\begin{array}{lllll}
x_{4} & x_{5} & x_{4} x_{5} & x_{4} x_{5}^{2} & 1
\end{array}\right)^{t}=0,
$$


where $C \in \mathbb{R}^{2 \times 5}$ is the coefficient matrix:

$$
C=\left(\begin{array}{ccccc}
1 & 0 & C_{13} & C_{14} & -T_{1} \\
0 & 1 & C_{23} & C_{24} & -T_{2}
\end{array}\right)
$$

and $C_{13}=k_{5}\left(\frac{1}{k_{2}}+\frac{1}{k_{3}}\right), C_{14}=\frac{k_{4} k_{5}}{k_{3}}\left(\frac{1}{k_{1}}+\frac{1}{k_{2}}\right), C_{23}=\frac{k_{5}}{k_{6}}$ and $C_{24}=\frac{k_{4} k_{5}}{k_{3} k_{6}}$. If we order the variables $\left(x_{4}, x_{5}\right)$ the support of this system is:

$$
\mathcal{A}=\{(1,0),(0,1),(1,1),(1,2),(0,0)\} .
$$

We depict in Figure 4 the 2-simplices $\Delta_{1}=\{(1,0),(1,1),(0,0)\}, \Delta_{2}=\{(1,1),(1,2),(0,0)\}$ and $\Delta_{3}=\{(0,1),(1,2),(0,0)\}$, which form a regular triangulation $\Gamma$ of $\mathcal{A}$, associated for instance with any height function $h: \mathcal{A} \rightarrow \mathbb{R}$ satisfying $h(1,0)=h_{1}, h(0,1)=h_{2}$, $h(1,1)=0, h(1,2)=0$, and $h(0,0)=0$, with $h_{1}, h_{2}>0$.
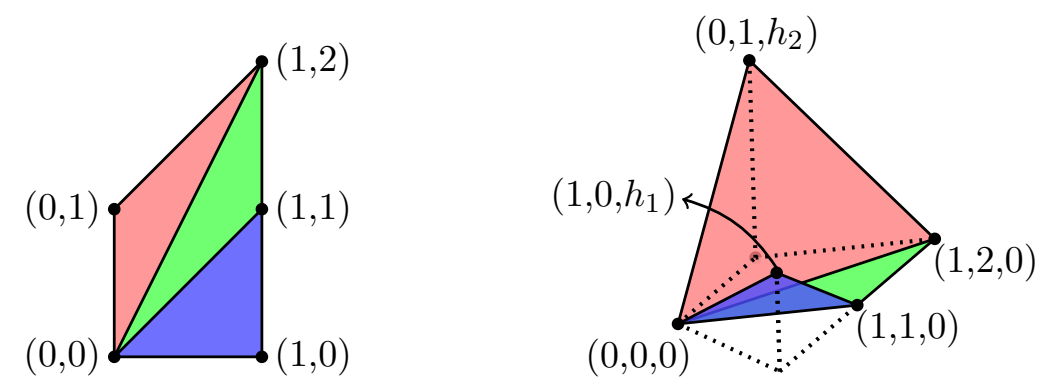

Figure 4. A regular triangulation $\Gamma$ of $\mathcal{A}$.

The simplex $\Delta_{1}$ is positively decorated by $C$ if and only if

$$
T_{1} k_{2} k_{3}-T_{2} k_{2} k_{6}-T_{2} k_{3} k_{6}>0,
$$

and the simplex $\Delta_{3}$ is positively decorated by $C$ if and only if

$$
T_{1} k_{1} k_{2}-T_{2} k_{1} k_{6}-T_{2} k_{2} k_{6}<0 \text {. }
$$

If conditions (2.13) and (2.14) hold, then the simplex $\Delta_{2}$ is also positively decorated by $C$ if and only if $k_{1}<k_{3}$. So, the three simplices are positively decorated by $C$ under the validity of condition (1.4):

$$
k_{6}\left(\frac{1}{k_{2}}+\frac{1}{k_{3}}\right)<\frac{T_{1}}{T_{2}}<k_{6}\left(\frac{1}{k_{1}}+\frac{1}{k_{2}}\right) .
$$

Assume that both inequalities in (1.4) hold. In this case, Theorem 2.9 says that there exists $t_{0} \in \mathbb{R}_{>0}$ such that for all $0<t<t_{0}$, the system

$$
\begin{aligned}
& t^{h_{1}} x_{4}+C_{13} x_{4} x_{5}+C_{14} x_{4} x_{5}^{2}-T_{1}=0, \\
& t^{h_{2}} x_{5}+C_{23} x_{4} x_{5}+C_{24} x_{4} x_{5}^{2}-T_{2}=0,
\end{aligned}
$$

has at least three positive nondegenerate solutions.

If we make the change of variables: $\bar{x}_{4}=t^{h_{1}} x_{4}, \bar{x}_{5}=t^{h_{2}} x_{5}$ we have:

$$
\begin{aligned}
& \bar{x}_{4}+t^{-\left(h_{1}+h_{2}\right)} C_{13} \bar{x}_{4} \bar{x}_{5}+t^{-\left(h_{1}+2 h_{2}\right)} C_{14} \bar{x}_{4} \bar{x}_{5}^{2}-T_{1}=0, \\
& \bar{x}_{5}+t^{-\left(h_{1}+h_{2}\right)} C_{23} \bar{x}_{4} \bar{x}_{5}+t^{-\left(h_{1}+2 h_{2}\right)} C_{24} \bar{x}_{4} \bar{x}_{5}^{2}-T_{2}=0 .
\end{aligned}
$$

If we consider the rescalings:

$$
\overline{k_{4}}=t^{-h_{2}} k_{4}, \quad \overline{k_{5}}=t^{-\left(h_{1}+h_{2}\right)} k_{5},
$$

and we keep fixed the values of the remaining constants $k_{1}, k_{2}, k_{3}, k_{6}$ and the total concentrations $T_{1}, T_{2}$, then the steady states of the dynamical system associated with the network with these rate and total conservation constants are the solutions of the polynomial system (2.16). And then, for these constants the network has at least three positive steady states. If we take $N_{1}=t_{0}^{-h_{2}}$ and $N_{2}=t_{0}^{-h_{1}}$ and we consider any positive $\beta_{4}, \beta_{5}$ satisfying 
$\beta_{4}>N_{1}$ and $\frac{\beta_{5}}{\beta_{4}}>N_{2}$, there exist $0<t<t_{0}$ such that $\beta_{4}=t^{-h_{1}}$ and $\beta_{5}=t^{-\left(h_{1}+h_{2}\right)}$ and we are done.

Another way to finish the proof of Theorem 1.1 is using Theorem 2.11. The inequalities that define the cone $\mathcal{C}_{\Gamma}$ are: $\left\langle m_{1}, h\right\rangle>0,\left\langle m_{2}, h\right\rangle>0$, where $m_{1}=(1,0,-2,1,0)$ and $m_{2}=(0,1,1,-1,-1)$. Fix $\varepsilon \in(0,1)^{2}$. As (1.4) holds, Theorem 2.11 says that there exist $M_{1}=M_{1}(\varepsilon), M_{2}=M_{2}(\varepsilon)>0$ such that the polynomial system

$$
\begin{aligned}
& \gamma_{1} x_{4}+\gamma_{3} C_{13} x_{4} x_{5}+\gamma_{4} C_{14} x_{4} x_{5}^{2}-\gamma_{5} T_{1}=0, \\
& \gamma_{2} x_{5}+\gamma_{3} C_{23} x_{4} x_{5}+\gamma_{4} C_{24} x_{4} x_{5}^{2}-\gamma_{5} T_{2}=0,
\end{aligned}
$$

has at least three nondegenerate positive solutions for any vector $\gamma \in\left(\mathbb{R}_{>0}\right)^{5}$ satisfying $\gamma^{m_{1}}<M_{1}$ and $\gamma^{m_{2}}<M_{2}$. In particular, this holds if we take $\gamma_{1}=\gamma_{2}=\gamma_{5}=1$, and $\gamma_{3}$ and $\gamma_{4}$ satisfy:

$$
\gamma_{3}^{-2} \gamma_{4}<M_{1}, \quad \gamma_{3} \gamma_{4}^{-1}<M_{2} .
$$

If we call $\beta_{4}=\frac{\gamma_{4}}{\gamma_{5}}, \beta_{5}=\gamma_{5}, N_{1}=\frac{1}{M_{1}}, N_{2}=\frac{1}{M_{2}}$, the inequalities in (2.18) are equivalent to $\beta_{4}>N_{1}$ and $\frac{\beta_{5}}{\beta_{4}}>N_{2}$. Then, if $\beta_{4}$ and $\beta_{5}$ satisfy these bounds, rescaling of the given parameters $k_{4}, k_{5}$ by $\overline{k_{4}}=\beta_{4} k_{4}, \overline{k_{5}}=\beta_{5} k_{5}$, gives raise to a multistationary dynamical system, as we claimed.

\section{THE MIXED APPROACH}

In this section, we present a similar but different approach to Theorems 2.9 and 2.11 . As the polynomials $f_{1}, \ldots, f_{d}$ might have different supports $\mathcal{A}_{1}, \ldots, \mathcal{A}_{d} \subset \mathbb{Z}^{d}$, one usually takes the union of the supports $\mathcal{A}=\cup_{i=1}^{d} \mathcal{A}_{i}$. That is, we can write the polynomial system

$$
f_{i}(x)=\sum_{a_{j} \in \mathcal{A}_{i}} c_{i, j} x^{a_{j}} \in \mathbb{R}\left[x_{1}, \ldots, x_{d}\right], i=1, \ldots, d
$$

in the form

$$
f_{i}(x)=\sum_{a_{j} \in \mathcal{A}} c_{i, j} x^{a_{j}} \in \mathbb{R}\left[x_{1}, \ldots, x_{d}\right], i=1, \ldots, d,
$$

where $c_{i, j}=0$ in case $a_{j} \notin \mathcal{A}_{i}$.

If one takes the same height function from $\mathcal{A}$ to $\mathbb{R}$ for each equation, the coefficient matrix $C$ might have many zero minors. Thus, we now allow different height functions $h^{(i)}: \mathcal{A}_{i} \rightarrow \mathbb{R}, i=1, \ldots, d$. Instead of considering regular subdivisions of $\mathcal{A}$, we will consider regular mixed subdivisions of the Minkowski sum $\mathcal{M}=\sum_{i=1}^{d} \mathcal{A}_{i}$ defined by height functions $h^{(i)}: \mathcal{A}_{i} \rightarrow \mathbb{R}, i=1, \ldots, d$. The projection of the lifted points in each of the faces of the lower convex hull of the Minkowski sum $\sum_{i=1}^{d} \mathcal{A}^{h^{(i)}}$ of the lifted point sets $\mathcal{A}^{h^{(i)}} \subset \mathbb{R}^{d+1}$ defines the associated regular mixed subdivision $S_{h}$ of $\mathcal{M}$. The convex hull of the cells in $S_{h}$ do not intersect or the intersection is a common face.

Regular mixed subdivisions of $\mathcal{M}$ are in bijection with regular subdivisions of the associated Cayley configuration $C\left(\mathcal{A}_{1}, \ldots, \mathcal{A}_{d}\right)$. This is the lattice configuration in $\mathbb{Z}^{d} \times \mathbb{Z}^{d}$ defined by

$$
C\left(\mathcal{A}_{1}, \ldots, \mathcal{A}_{d}\right)=\left(\mathcal{A}_{1} \times\left\{e_{1}\right\}\right) \cup \cdots\left(\mathcal{A}_{d-1} \times\left\{e_{d-1}\right\}\right) \cup\left(\mathcal{A}_{d} \times\left\{e_{d}\right\}\right),
$$

where $e_{1}, \ldots, e_{d}$ denotes the canonical basis in $\mathbb{Z}^{d}$. This is the support of the Cayley polynomial:

$$
F(x, y)=\sum_{i=1}^{d} y_{i} f_{i}(x),
$$

in variables $\left(x_{1}, \ldots, x_{d}, y_{1}, \ldots, y_{d}\right)$, associated with polynomials $f_{i}(x)$ with support in $\mathcal{A}_{i}$, $i=1, \ldots, d$. 
Note that the sum of the last $d$ coordinates of any point in $C\left(\mathcal{A}_{1}, \ldots, \mathcal{A}_{d}\right)$ equals 1 , so the maximal dimension of a simplex in the Cayley configuration is $2 d-1$ and then this simplex consists of $2 d$ points. We will assume that $C\left(\mathcal{A}_{1}, \ldots, \mathcal{A}_{d}\right)$ contains a $(2 d-1)$-simplex.

A tuple of height functions $\left(h^{(1)}, \ldots, h^{(d)}\right)$ as above can be identified with a height function $h: C\left(\mathcal{A}_{1}, \ldots, \mathcal{A}_{d}\right) \rightarrow \mathbb{R}$, defining $h\left(a_{j}, e_{i}\right)=h^{(i)}\left(a_{j}\right), i=1, \ldots, d$. In case $\Delta$ is a $(2 d-1)$-simplex in the associated regular subdivision $\Gamma_{h}$ of $C\left(\mathcal{A}_{1}, \ldots, \mathcal{A}_{d}\right)$, necessarily $\Delta$ contains at least one point $\left(a_{j}, e_{i}\right)$ in each $\mathcal{A}_{i}$. The corresponding maximal cell in the associated regular subdivision $S_{h}$ of $\mathcal{M}$ consists of all points of the form $b_{1}+\cdots+b_{d}$ with $\left(b_{i}, e_{i}\right)$ in $\Delta$. For more details about the translation between regular subdivisions of $C\left(\mathcal{A}_{1}, \ldots, \mathcal{A}_{d}\right)$ and regular mixed subdivisions of $\mathcal{M}$, we refer to Section 9.2 in [9]. We show this correspondence in Example 3.4 below.

Definition 3.1. $A(2 d-1)$-simplex $\Delta$ in the Cayley configuration $C\left(\mathcal{A}_{1}, \ldots, \mathcal{A}_{d}\right)$ is said to be mixed if it consists of two points $\left(a_{j_{1}}, e_{i}\right),\left(a_{j_{2}}, e_{i}\right)$ for each $i=1, \ldots, d$, with $a_{j_{1}}, a_{j_{2}} \in$ $\mathcal{A}_{i}$. A mixed simplex $\Delta$ is said to be positively decorated by $C$ if for each $i=1, \ldots, d$, the coefficients of the polynomial $f_{i}$ corresponding to the monomials $a_{j_{1}}$ and $a_{j_{2}}$ have different signs, that is, if $c_{i, j_{1}} c_{i, j_{2}}<0$.

Let $\Gamma$ be a regular subdivision of the Cayley configuration $C\left(\mathcal{A}_{1}, \ldots, \mathcal{A}_{d}\right)$. Let $h$ be a height vector that induces $\Gamma$ and denote by $h^{(1)}, \ldots, h^{(d)}$ the real vectors of size equal to the cardinality of $\mathcal{A}_{i}$, such that $h^{(i)}\left(a_{j}\right)=h\left(a_{j}, e_{i}\right)$, for $i=1, \ldots, d$, and $a_{j} \in \mathcal{A}_{i}$. Consider the family of polynomial systems parametrized by a positive real number $t$ :

$$
f_{i, t}(x)=\sum_{a_{j} \in \mathcal{A}_{i}} c_{i, j} t^{h^{(i)}\left(a_{j}\right)} x^{a_{j}} \in \mathbb{R}\left[x_{1}, \ldots, x_{d}\right], i=1, \ldots, d, t>0 .
$$

We then have:

Theorem 3.2. Let $\mathcal{A}_{1}, \ldots, \mathcal{A}_{d}$ be finite sets in $\mathbb{Z}^{d}$. Assume there are $p$ mixed $(2 d-1)$ simplices $\Delta_{1}, \ldots, \Delta_{p}$ which occur in a regular subdivision $\Gamma$ of $C\left(\mathcal{A}_{1}, \ldots, \mathcal{A}_{d}\right)$ and which are positively decorated by a matrix $C \in \mathbb{R}^{d \times n}$. Let $h$ be a height function inducing $\Gamma$ and $h^{(i)}, i=1, \ldots, d$, defined as before. Then, there exists $t_{0} \in \mathbb{R}_{>0}$ such that for all $0<t<t_{0}$, the number of (nondegenerate) solutions of (3.3) contained in the positive orthant is at least $p$. In particular, the result holds if $\Delta_{1}, \Delta_{2}$ are two mixed $(2 d-1)$ simplices of $C\left(\mathcal{A}_{1}, \ldots, \mathcal{A}_{d}\right)$ which share a facet.

Proof. Let $\Delta$ be a mixed $(2 d-1)$-simplex in $\Gamma$. Then it consists of $2 d$ points: two points $\left(a_{j_{1}}, e_{i}\right),\left(a_{j_{2}}, e_{i}\right)$ for each $i=1, \ldots, d$, with $a_{j_{1}}, a_{j_{2}} \in \mathcal{A}_{i}$. Consider the system (3.1) restricted to the binomials with exponents $a_{j_{1}}, a_{j_{2}}$ in each $f_{i}$. When $\Delta$ is positively decorated by $C$, we get a binomial system of equations equal to zero with coefficients of opposite signs:

$$
c_{i, j_{1}} x^{a_{j_{1}}}+c_{i, j_{2}} x^{a_{j_{2}}}=0, \quad i=1, \ldots, d .
$$

The positive solutions of this binomial system are in correspondence with the solutions of a system of a form:

$$
x^{M}=\beta,
$$

where $M \in \mathbb{R}^{d \times d}$ is the matrix with $i$-th row equal to $a_{j_{1}}-a_{j_{2}}$ for each $i=1, \ldots, d$, and $\beta=-\frac{c_{i, j_{2}}}{c_{i, j_{1}}} \in \mathbb{R}_{>0}^{d}$. Taking logarithms, we obtain the equivalent linear system:

$$
M^{t} \log (x)=\log (\beta),
$$

where $\log (x)=\left(\log \left(x_{1}\right), \ldots, \log \left(x_{d}\right)\right)$. As $\Delta$ is a maximal simplex, the matrix $M$ is invertible. Then, the linear system (3.4) has a solution, and thus the binomial system has a positive solution. Therefore, for each positively decorated simplex $\Delta$ with vertices $\left(a_{j_{1}}, e_{i}\right),\left(a_{j_{2}}, e_{i}\right)$, for each $i$, system (3.1) restricted to the monomials with exponents $a_{j_{1}}, a_{j_{2}}$ in each $f_{i}$ has a solution in $\mathbb{R}_{>0}^{d}$. The rest of the proof follows from the arguments in the proof of Theorem 3.4 in [2].

Furthermore, with a similar proof as Theorem 2.11, we have: 
Theorem 3.3. Let $\mathcal{A}_{1}, \ldots, \mathcal{A}_{d}$ be finite sets in $\mathbb{Z}^{d}$. Assume there exist $p$ mixed $(2 d-$ 1)-simplices $\Delta_{1}, \ldots, \Delta_{p}$ in $C\left(\mathcal{A}_{1}, \ldots, \mathcal{A}_{d}\right)$, which are part of a regular subdivision (for instance, when $p=2$ and the two simplices share a facet) and are positively decorated by $C$. Set $N=\left|\mathcal{A}_{1}\right|+\ldots\left|\mathcal{A}_{d}\right|$. Assume that the cone $\mathcal{C}_{\Delta_{1}, \ldots, \Delta_{p}}$ of all height vectors $h$ inducing regular subdivisions of $C\left(\mathcal{A}_{1}, \ldots, \mathcal{A}_{d}\right)$ containing $\Delta_{1}, \ldots, \Delta_{p}$ is defined by

$$
\mathcal{C}_{\Delta_{1}, \ldots, \Delta_{p}}=\left\{h \in \mathbb{R}^{N}:\left\langle m_{r}, h\right\rangle>0, r=1, \ldots, \ell\right\},
$$

where $m_{r}=\left(m_{r, 1}, \ldots, m_{r, N}\right) \in \mathbb{R}^{N}$.

Then, for any $\varepsilon \in(0,1)^{\ell}$ there exists $t_{0}(\varepsilon)>0$ such that for any $\gamma$ in the set

$$
U=\cup_{\varepsilon \in(0,1)^{\ell}}\left\{\gamma=\left(\gamma^{1}, \ldots, \gamma^{d}\right) \in \mathbb{R}_{>0}^{N}, ; \gamma^{m_{r}} \leq t_{0}(\varepsilon)^{\varepsilon_{r}}, r=1 \ldots, \ell\right\},
$$

the system

$$
\sum_{a_{j} \in \mathcal{A}_{i}} c_{i j} \gamma_{j}^{i} x^{a_{j}}=0, \quad i=1, \ldots, d,
$$

has at least $p$ nondegenerate solutions in the positive orthant, where $\gamma^{i}$ is a vector of size $\left|\mathcal{A}_{i}\right|$ with coordinates $\gamma_{j}^{i}$, with $a_{j} \in \mathcal{A}_{i}$.

We now present an application of the mixed approach in Theorem 3.3 to the previous example of the two component system with Hybrid Histidine Kinase (1.2).

Example 3.4. Recall that we are looking for positive solutions of the system:

$$
C\left(\begin{array}{lllll}
x_{4} & x_{5} & x_{4} x_{5} & x_{4} x_{5}^{2} & 1
\end{array}\right)^{t}=0,
$$

where $C \in \mathbb{R}^{2 \times 5}$ is the coefficient matrix:

$$
C=\left(\begin{array}{ccccc}
1 & 0 & C_{13} & C_{14} & -T_{1} \\
0 & 1 & C_{23} & C_{24} & -T_{2}
\end{array}\right)
$$

with $C_{13}=k_{5}\left(\frac{1}{k_{2}}+\frac{1}{k_{3}}\right), C_{14}=\frac{k_{4} k_{5}}{k_{3}}\left(\frac{1}{k_{1}}+\frac{1}{k_{2}}\right), C_{23}=\frac{k_{5}}{k_{6}}$ and $C_{24}=\frac{k_{4} k_{5}}{k_{3} k_{6}}$.

The supports of the first and second polynomials are $\mathcal{A}_{1}=\{(1,0),(1,1),(1,2),(0,0)\}$ and $\mathcal{A}_{2}=\{(0,1),(1,1),(1,2),(0,0)\}$ respectively. We want to find mixed positively decorated mixed 3 -simplices of the Cayley configuration $C\left(\mathcal{A}_{1}, \mathcal{A}_{2}\right)$ occuring in a regular subdivision. As we mentioned, these mixed 3-simplices correspond to maximal dimension 2 mixed cells of the associated mixed subdivision of the Minkowski sum $\mathcal{A}_{1}+\mathcal{A}_{2}$ (Fig. 5).

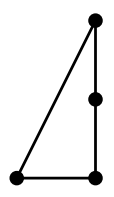

$\mathcal{A}_{1}$

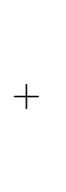<smiles>C1CCC1</smiles>

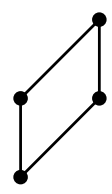

$\mathcal{A}_{2}$

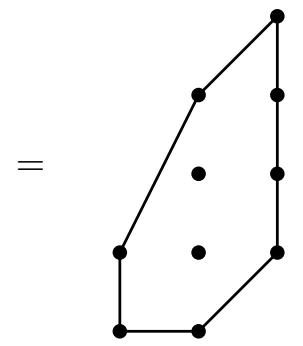

Figure 5. The Minkowski sum $\mathcal{M}=\mathcal{A}_{1}+\mathcal{A}_{2}$.

We can choose the following mixed 3 -simplices with vertices in $C\left(\mathcal{A}_{1}, \mathcal{A}_{2}\right)$ :

$$
\begin{aligned}
& \Delta_{1}=\left\{\left(0,0, e_{1}\right),\left(1,2, e_{1}\right),\left(0,0, e_{2}\right),\left(0,1, e_{2}\right)\right\}, \\
& \Delta_{2}=\left\{\left(0,0, e_{1}\right),\left(1,2, e_{1}\right),\left(0,0, e_{2}\right),\left(1,1, e_{2}\right)\right\}, \\
& \Delta_{3}=\left\{\left(0,0, e_{1}\right),\left(1,0, e_{1}\right),\left(0,0, e_{2}\right),\left(1,1, e_{2}\right)\right\},
\end{aligned}
$$

which are positively decorated by $C$. These simplices $\Delta_{1}, \Delta_{2}$ and $\Delta_{3}$ are in correspondence, respectively, with the mixed cells $\sigma_{1}=\{((0,0)=(0,0)+(0,0),(1,2)=(1,2)+$ $(0,0),(0,1)=(0,0)+(0,1),(1,3)=(1,2)+(0,1))\}, \sigma_{2}=\{((0,0),(1,2),(1,1),(2,3))\}$ and $\sigma_{3}=\{((0,0),(1,0),(1,1),(2,1))\}$, depicted in Figure 6 . 

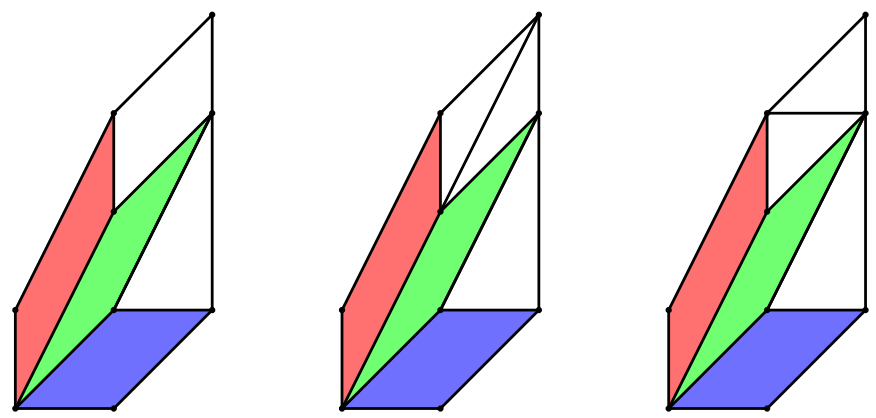

Figure 6 . Three regular mixed subdivisions of $\mathcal{M}$ that contain the mixed cells $\sigma_{1}, \sigma_{2}$ and $\sigma_{3}$.

The cone $\mathcal{C}_{\Delta_{1}, \Delta_{2}, \Delta_{3}}$ of height vectors $h=\left(h_{1}, \ldots, h_{8}\right) \in \mathbb{R}^{8}$ inducing regular subdivisions of $C\left(\mathcal{A}_{1}, \mathcal{A}_{2}\right)$ containing $\Delta_{1}, \Delta_{2}$ and $\Delta_{3}$ is defined by the inequalities $\left\langle m_{i}, h\right\rangle>0, i=$ $1, \ldots, 8$, where

$$
\begin{aligned}
& m_{1}=(1,0,-1,0,2,0,0,-2), m_{2}=(0,1,-1,0,1,0,0,-1), m_{3}=(0,0,-1,1,0,0,1,-1), \\
& m_{4}=(0,0,-1,1,1,1,0,-2), m_{5}=(1,0,1,-2,0,-2,0,2), m_{6}=(0,1,0,-1,0,-1,0,1), \\
& m_{7}=(1,0,0,-1,1,-1,0,0), m_{8}=(1,0,0,-1,0,-2,1,1),
\end{aligned}
$$

with $h_{1}=h\left(1,0, e_{1}\right), h_{2}=h\left(1,1, e_{1}\right), h_{3}=h\left(1,2, e_{1}\right), h_{4}=h\left(0,0, e_{1}\right), h_{5}=h\left(0,1, e_{2}\right)$, $h_{6}=h\left(1,1, e_{2}\right), h_{7}=h\left(1,2, e_{2}\right)$ and $h_{8}=h\left(0,0, e_{2}\right)$.

Fix $\varepsilon \in(0,1)^{8}$. Theorem 3.3 says that there exist positive constants $M_{i}=M_{i}(\varepsilon)$, $i=1, \ldots, 8$ such that the number of positive nondegenerate solutions of the polynomial system

$$
\begin{aligned}
& \gamma_{1}^{1} x_{4}+\gamma_{2}^{1} C_{13} x_{4} x_{5}+\gamma_{3}^{1} C_{14} x_{4} x_{5}^{2}-\gamma_{4}^{1} T_{1}=0, \\
& \gamma_{1}^{2} x_{5}+\gamma_{2}^{2} C_{23} x_{4} x_{5}+\gamma_{3}^{2} C_{24} x_{4} x_{5}^{2}-\gamma_{4}^{2} T_{2}=0,
\end{aligned}
$$

is at least the number of mixed positively decorated simplices, in this case 3 , for any vector $\gamma=\left(\gamma_{1}^{1}, \gamma_{2}^{1}, \gamma_{3}^{1}, \gamma_{4}^{1}, \gamma_{1}^{2}, \gamma_{2}^{2}, \gamma_{3}^{2}, \gamma_{4}^{2}\right) \in \mathbb{R}_{>0}^{8}$ that satisfies $\gamma^{m_{i}}<M_{i}$, for each $i=1, \ldots, 8$.

In particular we have the following result:

Proposition 3.5. Given positive reactions constants $k_{1}, \ldots, k_{6}$ and positive total conservations constants $T_{1}$ and $T_{2}$, there exist positive constants $N_{1}, N_{2}, N_{3}$ and $N_{4}$ such that for any $\beta_{1}, \beta_{2}>0$ satisfying

$$
N_{1}<\beta_{1}, \quad N_{2}<\beta_{2}, \quad \frac{\beta_{2}}{\beta_{1}}<N_{3}, \quad \frac{\beta_{1}}{\left(\beta_{2}\right)^{2}}<N_{4},
$$

the dynamical system corresponding to the Hybrid Histidine Kinase network (1.2) has at least 3 positive steady states, after replacing $k_{1}$ by $\bar{k}_{1}=\left(\beta_{1}\left(\frac{1}{k_{1}+k_{2}}\right)-\frac{1}{k_{2}}\right)^{-1}$ and rescaling $\bar{k}_{6}=\left(\beta_{2}\right)^{-1} k_{6}$, without altering the value of the other reaction and total conservation constants.

Proof. Take any positive vector $\gamma=\left(\gamma_{1}^{1}, \gamma_{2}^{1}, \gamma_{3}^{1}, \gamma_{4}^{1}, \gamma_{1}^{2}, \gamma_{2}^{2}, \gamma_{3}^{2}, \gamma_{4}^{2}\right)$ satisfying $\gamma_{1}^{1}=\gamma_{2}^{1}=$ $\gamma_{4}^{1},=\gamma_{1}^{2}=\gamma_{4}^{2}=1$, and $\gamma_{2}^{2}=\gamma_{3}^{2}$. Call $\beta_{1}=\gamma_{3}^{1}$ and $\beta_{2}=\gamma_{2}^{2}$. Then if $\beta_{1}, \beta_{2}$ satisfy

$$
N_{1}<\beta_{1}, \quad N_{2}<\beta_{2}, \quad \frac{\beta_{2}}{\beta_{1}}<N_{3}, \quad \frac{\beta_{1}}{\left(\beta_{2}\right)^{2}}<N_{4},
$$

where $N_{1}=\left(\min \left\{M_{1}, M_{2}, \frac{k_{2}}{k_{2}+k_{1}}\right\}\right)^{-1}, N_{2}=\left(\min \left\{M_{6}, M_{7}, M_{8}\right\}\right)^{-1}, N_{3}=\min \left\{M_{3}, M_{4}\right\}$ and $N_{4}=M_{5}$, the system

$$
\begin{aligned}
x_{4}+C_{13} x_{4} x_{5}+\beta_{1} C_{14} x_{4} x_{5}^{2}-T_{1}= & 0, \\
x_{5}+\beta_{2} C_{23} x_{4} x_{5}+\beta_{2} C_{24} x_{4} x_{5}^{2}-T_{2}= & 0,
\end{aligned}
$$

has at least 3 positive solutions. Returning to the original constants, if we keep fixed $k_{2}, k_{3}, k_{4}, k_{5}, T_{1}, T_{2}$ and we replace $k_{1}, k_{6}$ by $\bar{k}_{1}=\left(\beta_{1}\left(\frac{1}{k_{1}+k_{2}}\right)-\frac{1}{k_{2}}\right)^{-1}$ and $\bar{k}_{6}=\left(\beta_{2}\right)^{-1} k_{6}$, 
the positive steady states arising from the network with these constants are the positive solutions of the polynomial system (3.7), and so it is multistationary because there are at least 3 positive steady states in a fixed stoichiometric compatibility class. Observe that $k_{1}$ is positive because of the choice of $N_{1}$.

Notice that if the value of $\beta_{1}$ in the proof of Proposition 3.5 is large enough, $\bar{k}_{1}$ is smaller than $k_{3}$, the necessary and sufficient condition to guarantee multistationarity that appears in $[3]$.

\section{Distributive multisite PhOSPhORYLation SYSTEMS}

Phosphorylation/dephosphorylation are post-translational modification of proteins mediated by enzymes, particular proteins that add or take off a phosphate group at a specific site, inducing a conformational change that allows/prevents the protein to perform its function. The standard building block in cell signaling is the following enzyme mechanism, which is called a Michaelis-Menten mechanism.

$$
S_{0}+E \underset{k_{\text {off }}}{\stackrel{k_{\text {on }}}{\rightleftarrows}} E S_{0} \stackrel{k_{\text {cat }}}{\longrightarrow} S_{1}+E
$$

This basic network involves four species: the substrate $S_{0}$, the phosphorylated substrate $S_{1}$, the enzyme $E$, called kinase, and the intermediate species $E S_{0}$, and 3 reactions, with reaction constants called $k_{\text {on }}, k_{\text {off }}, k_{\text {cat }}$. The enzyme $E$ is not consumed after the whole mechanism, which is assumed to be with mass-action kinetics. The concentration of the donor of the phosphate group is considered to be constant, thus hidden in the reaction constants and ignored. This mechanism with 4 species, 3 complexes and 3 reactions is usually represented by the scheme depicted in Figure 7 .

$$
S_{0} \stackrel{E}{ }_{S_{1} .}
$$

FiguRE 7. The network (4.1).

The addition of phosphate groups to multiple sites of a single molecule, may be distributive or processive. Distributive systems require an enzyme and substrate to bind several times in order to add/remove multiple phosphate groups. Processive systems require only one binding to add/remove all phosphate groups and it was shown in [6] that such systems cannot admit more than one steady state in each stoichiometric compatibility class. The distibutive multisite phosphorylation system describes the $n$-site phosphorylation of a protein by a kinase/phosphatase pair in a sequential and distributive mechanism and it is known that it has the capacity of multistationarity for any $n \geq 2$ [28].

The reaction mechanism for the sequential distributive mechanism for the $n$-site network is a sequence of reactions as in (4.1), where we append $n$ subgraphs of the form:

$$
S_{i}+E \underset{k_{\mathrm{off}_{\mathrm{i}}}}{\stackrel{k_{\mathrm{on}_{\mathrm{i}}}}{\rightleftarrows}} E S_{i} \stackrel{k_{\mathrm{cat}_{\mathrm{i}}}}{\rightarrow} S_{i+1}+E, i=0, \ldots, n-1,
$$

and, on the other side, $n$ subgraphs of the form:

$$
S_{i}+F \underset{\ell_{\mathrm{off}_{\mathrm{i}-1}}}{\stackrel{\ell_{\mathrm{on}_{\mathrm{i}}-1}}{\rightleftarrows}} F S_{i} \stackrel{\ell_{\mathrm{cat}_{\mathrm{i}}-1}}{\rightarrow} S_{i-1}+F, i=1, \ldots, n,
$$


where $F$ denotes another enzyme called phosphatase, to obtain the network:

$$
\begin{aligned}
& S_{0}+E \underset{k_{\mathrm{off}_{0}}}{\stackrel{k_{\mathrm{on}_{0}}}{\rightleftarrows}} E S_{0} \stackrel{k_{\mathrm{cat}_{0}}}{\rightarrow} S_{1}+E \cdots \rightarrow S_{n-1}+E \underset{k_{\mathrm{off}_{\mathrm{n}}}}{\stackrel{k_{\mathrm{on}_{\mathrm{n}}-1}}{\rightleftarrows}} E S_{n-1} \stackrel{k_{\mathrm{cat}_{\mathrm{n}}-1}}{\rightarrow} S_{n}+E \\
& S_{n}+F \underset{\ell_{\mathrm{off}_{\mathrm{n}}-1}}{\stackrel{\ell_{\mathrm{on}_{\mathrm{n}}-1}}{\rightleftarrows}} F S_{n} \stackrel{\ell_{\mathrm{cat}_{\mathrm{n}}-1}}{\rightarrow} S_{n-1}+F \cdots \rightarrow S_{1}+F \underset{\ell_{\mathrm{off}_{0}}}{\stackrel{\ell_{\mathrm{on}_{0}}}{\rightleftarrows}} F S_{1} \stackrel{\ell_{\text {cat }}}{\rightarrow} S_{0}+F
\end{aligned}
$$

It represents one substrate that can sequentially acquire up to $n$ phosphate groups, via the action of the kinase $E$, and which can be sequentially released via the action of the phosphatase $F$, in both cases via an intermediate species formed by the union of the substrate and the enzyme. The kinetics of this network is deduced by applying the law of mass action to this explicit labeled digraph. There are $3 n+3$ species: the substrate species $S_{0}, S_{1}, \ldots, S_{n}$, the enzyme species $E$ and $F$ and the intermediate species $E S_{0}$, $E S_{1}, \ldots, E S_{n-1}, F S_{1}, F S_{2}, \ldots, F S_{n}$. We denote by $s_{0}, s_{1}, \ldots, s_{n}, e, f, y_{0}, y_{1}, \ldots, y_{n-1}$, $u_{0}, u_{1}, \ldots, u_{n-1}$ the concentration of the species $S_{0}, S_{1}, \ldots, S_{n}, E, F, E S_{0}, E S_{1}, \ldots$, $E S_{n-1}, F S_{1}, F S_{2}, \ldots, F S_{n}$ respectively. The associated dynamical system that arises under mass-action kinetics equals:

$$
\begin{aligned}
\frac{d s_{0}}{d t} & =-k_{\mathrm{on}_{0}} s_{0} e+k_{\mathrm{off}_{0}} y_{0}+\ell_{\mathrm{cat}_{0}} u_{0}, \\
\frac{d s_{i}}{d t} & =k_{\mathrm{cat}_{\mathrm{i}-1}} y_{i-1}-k_{\mathrm{on}_{\mathrm{i}}} s_{i} e+k_{\mathrm{off}_{\mathrm{i}}} y_{i}+\ell_{\mathrm{cat}_{\mathrm{i}}} u_{i}-\ell_{\mathrm{on}_{\mathrm{i}-1}} s_{i} f+\ell_{\mathrm{off}_{\mathrm{i}-1}} u_{i-1}, i=1, \ldots, n-1, \\
\frac{d s_{n}}{d t} & =k_{\mathrm{cat}_{\mathrm{n}-1}} y_{n-1}-\ell_{\mathrm{on}_{\mathrm{n}-1}} s_{n} f+\ell_{\mathrm{off}_{\mathrm{n}-1}} u_{n-1}, \\
\frac{d y_{i}}{d t} & =k_{\mathrm{on}_{\mathrm{i}}} s_{i} e-\left(k_{\mathrm{off}_{\mathrm{i}}}+k_{\mathrm{cat}_{\mathrm{i}}}\right) y_{i}, i=0, \ldots, n-1, \\
\frac{d u_{i}}{d t} & =\ell_{\mathrm{on}_{\mathrm{i}}} s_{i+1} f-\left(\ell_{\mathrm{off}_{\mathrm{i}}}+\ell_{\mathrm{cat}_{\mathrm{i}}}\right) u_{i}, i=0, \ldots, n-1, \\
\frac{d e}{d t} & =-\sum_{i=0}^{n-1} \frac{d y_{i}}{d t}, \quad \frac{d f}{d t}=-\sum_{i=0}^{n-1} \frac{d u_{i}}{d t} .
\end{aligned}
$$

There are three linearly independent conservation laws for any value of $n$ (and no more):

$$
\sum_{i=0}^{n} s_{i}+\sum_{i=0}^{n-1} y_{i}+\sum_{i=0}^{n-1} u_{i}=S_{t o t}, \quad e+\sum_{i=0}^{n-1} y_{i}=E_{t o t}, \quad f+\sum_{i=0}^{n-1} u_{i}=F_{t o t},
$$

where clearly the total amounts $S_{t o t}, E_{t o t}, F_{t o t} \in \mathbb{R}_{>0}$ for any trajectory intersecting the positive orthant. It is straigthforward to see from the differential equations that the concentrations of the intermediates species at steady state satisfy the following binomial equations:

$$
y_{i}-K_{i} e s_{i}=0, i=0, \ldots, n-1, \quad u_{i}-L_{i} f s_{i+1}=0, i=0, \ldots, n-1,
$$

where $K_{i}=\frac{k_{\mathrm{on}_{\mathrm{i}}}}{k_{\mathrm{off}_{\mathrm{i}}}+k_{\mathrm{cat}_{\mathrm{i}}}}$ and $L_{i}=\frac{\ell_{\mathrm{on}_{\mathrm{i}}}}{\ell_{\mathrm{off}_{\mathrm{i}}}+\ell_{\mathrm{cat}_{\mathrm{i}}}}$ for each $i=0, \ldots, n-1\left(K_{i}^{-1}\right.$ and $L_{i}^{-1}$ are usually called Michaelis-Menten constants, $i=0, \ldots, n-1)$.

Sequential phosphorylation mechanisms are an example of $s$-toric MESSI networks, defined in [24]. We recall in Section 5 their definition and we present general results for $s$-toric MESSI systems that explain our computations in this section. In particular, by Theorem 3.5 in [24] we can find the following binomial equations that describe the steady states. The whole steady state variety can be cut out in the positive orthant by adding to the binomials in (4.3), the binomial equations:

$$
\tau_{i} s_{i} e-\nu_{i} s_{i+1} f=0,
$$

where $\tau_{i}=k_{\text {cat }_{\mathrm{i}}} K_{i}$ and $\nu_{i}=\ell_{\text {cat }_{\mathrm{i}}} L_{i}$, for each $i=0, \ldots, n-1$. Using these binomial equations, we can parametrize the positive steady states by monomials. For instance, we 
can write the concentration at steady state of all species in terms of the species $s_{0}, e, f$ :

$$
\begin{array}{rll}
s_{i} & =T_{i-1} \frac{s_{0} e^{i}}{f^{i}}, & i=1, \ldots, n, \\
y_{i}= & K_{i} T_{i-1} \frac{s_{0} e^{i+1}}{f^{i}}, & i=0, \ldots, n-1, \\
u_{i}= & L_{i} T_{i} \frac{s_{0} e^{i+1}}{f^{i}}, & i=0, \ldots, n-1,
\end{array}
$$

where $T_{i}=\prod_{j=0}^{i} \frac{\tau_{j}}{\nu_{j}}$ for $i=0, \ldots, n-1$ and $T_{-1}=1$.

We will use this parametrization in order to apply Theorems 2.9 and 2.11 to the sequential phosphorylation mechanisms for any $n$ :

Theorem 4.1. With the previous notations, assume

$$
S_{t o t}>F_{t o t} .
$$

Then, there is a choice of rate constants for which the distributive n-site phosphorylation system is multistationary. More explicitly, for any choice of positive real numbers $k_{\mathrm{cat}_{1}}, \ell_{\mathrm{cat}_{1}}$ satisfying

$$
\frac{k_{\mathrm{cat}_{1}}}{\ell_{\mathrm{cat}_{1}}}>\max \left\{\frac{F_{t o t}}{S_{\text {tot }}-F_{\text {tot }}}, \frac{F_{t o t}}{E_{\text {tot }}}\right\},
$$

fix any value of the remaining rate constants and positive numbers $h_{i}$, for $i=4, \ldots, 2 n+3$ such that $i h_{n+5}<h_{i+3}$ for $i=1, \ldots, n$ and $(i-1) h_{n+5}<h_{n+i+3}$ for $i=1,3, \ldots, n$. Then, there exists $t_{0}>0$ such that for any value of $t \in\left(0, t_{0}\right)$ the system is multistationary after the rescalings $t^{h_{n+4}} k_{\mathrm{on}_{0}}, t^{h_{n+4+i}-h_{i+3}} k_{\mathrm{on}_{\mathrm{i}}}, i=1, \ldots, n-1, t^{h_{n+4+i}-h_{i+4}} \ell_{\mathrm{on}_{\mathrm{i}}}, i=0, \ldots, n-1$.

Similarly, for any fixed choice of reaction rate constants and total conservation constants satysfying (4.4) and (4.5), there exist positive constants $M_{i}, i=1, \ldots, 4 n-2$ such that for any positive values of $\gamma_{i}, i=1, \ldots, 2 n$ verifying

$$
\begin{aligned}
\gamma_{i} & <M_{i}, i=1, \ldots, 2 n, \quad \frac{\gamma_{i}}{\gamma_{n+2}^{i}}<M_{2 n+i}, i=1, \ldots, n, \\
\frac{\gamma_{n+i}}{\gamma_{n+2}^{i-1}} & <M_{3 n-2+i}, i=3, \ldots, n,
\end{aligned}
$$

the rescaling of the given parameters $k_{o n_{1}}, k_{o n_{i}}, i=2, \ldots, n-1, \ell_{o n_{i}}, i=1, \ldots, n-1$ by

$$
\gamma_{n+1} k_{o n_{1}}, \frac{\gamma_{n+1+i}}{\gamma_{i}} k_{o n_{i}}, i=2, \ldots, n-1, \frac{\gamma_{n+1+i}}{\gamma_{i+1}} \ell_{o n_{i}}, i=1, \ldots, n-1,
$$

respectively, gives raise to a multistationary system.

Proof. Previously in this section, we showed that we can write the concentration at steady state of all species in terms of the species $\left(s_{0}, e, f\right)$, as in (4). We substitute this monomial parametrization of the steady states into the linear conservation relations (4.2). We have a system of three equations and we write it in matricial form:

$$
C\left(\begin{array}{llllllllll}
s_{0} & e & f & s_{0} e f^{-1} & \ldots & s_{0} e^{n} f^{-n} & s_{0} e f^{0} & \ldots & s_{0} e^{n} f^{-(n-1)} & 1
\end{array}\right)^{t}=0,
$$

where the matrix $C \in \mathbb{R}^{3 \times(2 n+4)}$ is the matrix of coefficients:

$$
C=\left(\begin{array}{cccccccccc}
1 & 0 & 0 & T_{0} & \ldots & T_{n-1} & K_{0}+L_{0} T_{0} & \ldots & K_{n-1} T_{n-2}+L_{n-1} T_{n-1} & -S_{t o t} \\
0 & 1 & 0 & 0 & \ldots & 0 & K_{0} & \ldots & K_{n-1} T_{n-2} & -E_{t o t} \\
0 & 0 & 1 & 0 & \ldots & 0 & L_{0} T_{0} & \ldots & L_{n-1} T_{n-1} & -F_{t o t}
\end{array}\right)
$$

If we order the variables in this way: $s_{0}, e, f$, the support of the system is:

$$
\begin{array}{r}
\mathcal{A}=\{(1,0,0),(0,1,0),(0,0,1),(1,1,-1),(1,2,-2), \ldots,(1, n,-n), \\
(1,1,0),(1,2,-1), \ldots,(1, n,-(n-1)),(0,0,0)\} .
\end{array}
$$


We want to find two positively decorated 3 -simplices with vertices in $\mathcal{A}$ which share a facet. For example we take the simplices

$$
\begin{aligned}
& \Delta_{1}=\{(1,0,0),(0,1,0),(0,0,1),(0,0,0)\}, \\
& \Delta_{2}=\{(1,0,0),(0,1,0),(1,2,-1),(0,0,0)\} .
\end{aligned}
$$

They are shown in Figure 8, made with Polymake [15], which is a very useful tool to visualize and to do computations with polytopes and triangulations.

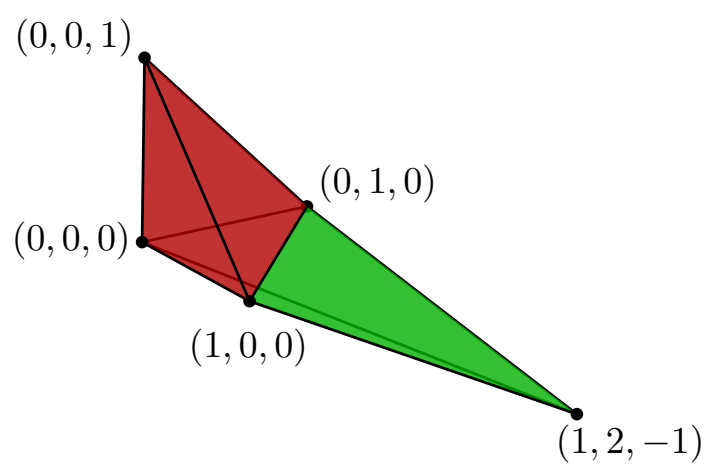

Figure 8 . The simplices $\Delta_{1}$ and $\Delta_{2}$.

The simplex $\Delta_{1}$ is automatically positively decorated by $C$. The simplex $\Delta_{2}$ is positively decorated by $C$ if and only if:

$$
E_{t o t}-\frac{K_{1} T_{0} F_{t o t}}{L_{1} T_{1}}>0, \quad \text { and } S_{t o t}-\frac{\left(K_{1} T_{0}+L_{1} T_{1}\right) F_{t o t}}{L_{1} T_{1}}>0
$$

Getting back to the original constants, we can write the previous conditions in the following form:

$$
\begin{gathered}
S_{t o t}>F_{\text {tot }}, \\
\frac{k_{\mathrm{cat}_{1}}}{\ell_{\mathrm{cat}_{1}}}>\max \left\{\frac{F_{t o t}}{S_{\text {tot }}-F_{\text {tot }}}, \frac{F_{t o t}}{E_{\text {tot }}}\right\} .
\end{gathered}
$$

Suppose that conditions (4.8) hold. Then the simplices $\Delta_{1}$ and $\Delta_{2}$ are positively decorated. Proposition 2.5 says that exists a regular triangulation of $\Gamma$ of the convex hull of $\mathcal{A}$, such that the two simplices $\Delta_{1}$ and $\Delta_{2}$ are part of that triangulation. Given any height function $h$ inducing such a $\Gamma$, by Theorem 2.9 there exists $t_{0} \in \mathbb{R}_{>0}$ such that for all $0<t<t_{0}$, the number of positive nondegenerate solutions of the scaled system:

$$
\begin{aligned}
& t^{h_{1}} s_{0}+\sum_{i=1}^{n} T_{i-1} t^{h_{i+3}} \frac{s_{0} e^{i}}{f^{i}}+\sum_{i=0}^{n-1}\left(K_{i} T_{i-1}+L_{i} T_{i}\right) t^{h_{n+4+i}} \frac{s_{0} e^{i+1}}{f^{i}}-S_{\text {tot }} t^{h_{2 n+4}}=0, \\
& t^{h_{2}} e+\sum_{i=0}^{n-1} K_{i} T_{i-1} t^{h_{n+4+i}} \frac{s_{0} e^{i+1}}{f^{i}}-E_{t o t} t^{h_{2 n+4}}=0, \\
& t^{h_{3}} f+\sum_{i=0}^{n-1} L_{i} T_{i} t^{h_{n+4+i}} \frac{s_{0} e^{i+1}}{f^{i}}-F_{t o t} t^{h_{2 n+4}}=0,
\end{aligned}
$$

is at least two, where $h_{1}=h(1,0,0), h_{2}=h(0,1,0), h_{3}=h(0,0,1), h_{i+3}=h(1, i,-i)$, for $i=1, \ldots, n, h_{n+3+i}=h(1, i,-(i-1))$, for $i=1, \ldots, n$ and $h_{2 n+4}=h(0,0,0)$.

We can suppose without loss of generality that $h_{1}=h_{2}=h_{3}=h_{2 n+4}=0$ and $h(1,2,-1)=h_{n+5}>0$. Let $\varphi_{1}$ and $\varphi_{2}$ be the affine linear functions $\varphi_{1}(x, y, z)=0$ and $\varphi_{2}(x, y, z)=-h_{n+5} z$ which agree with $h$ on the simplices $\Delta_{1}$ and $\Delta_{2}$ respectively. Then,

$0<h_{i+3}, \quad \varphi_{2}(1, i,-i)=h_{n+5} i<h_{i+3} \quad$ for $i=1, \ldots, n$,

$0<h_{n+3+i}, \quad \varphi_{2}(1, i,-(i-1))=h_{n+5}(i-1)<h_{n+3+i} \quad$ for $i=1,3 \ldots, n, i \neq 2$. 
Any such choice defines a regular subdivision containing both simplices (and if the heights are generic the subdivision is a regular triangulation).

If we rescale the following constants:

$$
\begin{aligned}
& t^{h_{n+4}} K_{0}, \quad t^{h_{n+4+i}-h_{i+3}} K_{i}, i=1, \ldots, n-1 \\
& t^{h_{n+4+i}-h_{i+4}} L_{i}, i=0, \ldots, n-1 .
\end{aligned}
$$

and we keep fixed the values of the constants $k_{\text {cat }_{1}}$ and $\ell_{\text {cat }_{1}}$ and the total values $E_{\text {tot }}, F_{\text {tot }}$ and $S_{\text {tot }}$ (such that (4.8) holds), the dynamical system obtained from the network with these constants is the system (4.9). And then, for these constants the network has at least two positive steady states. Moreover, it is straightforward to check that it is enough to rescale the following original constants as indicated in the statement:

$$
t^{h_{n+4}} k_{\mathrm{On}_{0}}, t^{h_{n+4+i}-h_{i+3}} k_{\mathrm{on}_{\mathrm{i}}}, i=1, \ldots, n-1, t^{h_{n+4+i}-h_{i+4}} \ell_{\mathrm{on}_{\mathrm{i}}}, i=0, \ldots, n-1,
$$

to get the equalities (4.10).

The last part of the statement follows with similar arguments via Proposition 2.10 and Theorem 2.11.

Remark 4.2. Using a parametrization of the concentrations of the species at steady state in terms of other variables (or with another choice of the simplices) we can obtain other regions in the parameters space that guarantee multistationarity .

\section{MESSI SYSTEMS}

In [24], Dickenstein and Pérez Millán introduced a general framework for biological systems, called MESSI systems, that describe Modifications of type Enzyme-Substrate or Swap with Intermediates. Distributive multisite phosphorylation systems and enzymatic cascades as the one we depict in Figure 9, with any number of layers which occur in cell signaling pathways (and we study in detail in [17]), are examples of MESSI systems of biological significance. In particular they are examples of $s$-toric MESSI systems, an important subclass of MESSI systems. The authors proved in [24] that any $s$-toric MESSI system is toric, that is, the positive steady states can be described with binomials and under certain hypotheses, they can choose explicit binomials with coefficients in $\mathbb{Q}(\kappa)$ which describe the positive steady states. Moreover, under certain combinatorial conditions, they describe a basis of conservation laws for these systems.

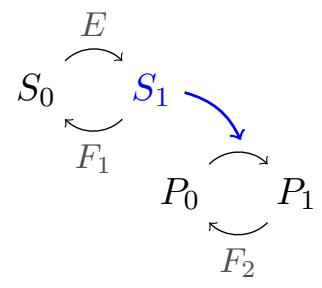

Figure 9. Scheme of a 2-layer cascade of GK-loops, similar to Fig. 7.

In order to apply our method in Section 2 to determine a multistationarity region for the network (1.2) or in Section 4 for the distributive multisite phosphorylation systems, we proposed and replaced a rational parametrization of the steady state variety into a basis of the conservation relations, and we then had to rescale some of original parameters at the end of the procedure (as in (4.11), (4.7)). Our main result in this section is Theorem 5.4, which guarantees that the rescaling of the parameters can be done for any $s$-toric MESSI system, together with Proposition 5.3 which ensures and describes the existence of a rational parametrization of the steady state variety. 
5.1. Basic definitions concerning MESSI systems. We briefly introduce the basic definitions of MESSI systems. For a more detailed explanation, see [24].

A MESSI network is a chemical reaction network, for which exists a partition of the set of species $\mathscr{S}$ into disjoint subsets:

$$
\mathscr{S}=\mathscr{S}^{(0)} \bigsqcup \mathscr{S}^{(1)} \bigsqcup \mathscr{S}^{(2)} \bigsqcup \cdots \bigsqcup \mathscr{S}^{(m)},
$$

where $m \geq 1$ and $\bigsqcup$ denotes disjoint union. Species in $\mathscr{S}^{(0)}$ are called intermediate and species in $\mathscr{S}_{1}:=\mathscr{S} \backslash \mathscr{S}^{(0)}$ are called core, with $\# \mathscr{S}^{(0)}=p$ and $\# \mathscr{S}^{(1)}=s-p>0$. As before, we denote the species with upper letters and the concentration of the species with small letters, for example $x_{j}$ denotes the concentration of the species $X_{j}$.

There are two types of complexes allowed in a MESSI network: intermediate complexes and core complexes. The intermediate complexes are complexes that consist of a unique intermediate species that only appears in that complex. The core complexes are mono or bimolecular and consist of either one or two core species. When a core complex consists of two species $X_{i}, X_{j}$, they must belong to different sets $\mathscr{S}^{(\alpha)}, \mathscr{S}^{(\beta)}$ with $\alpha \neq \beta, \alpha, \beta \geq 1$.

We say that a complex $y$ reacts to a complex $y^{\prime}$ via intermediates if either $y \rightarrow y^{\prime}$ or there exists a path of reactions from $y$ to $y^{\prime}$ only through intermediate complexes. This is denoted by $y \rightarrow_{\circ} y^{\prime}$. Another condition in the intermediate complexes is that for every intermediate complex $y$, there must exist core complexes $y_{1}$ and $y_{2}$ such that $y_{1} \rightarrow_{\circ} y$ and $y \rightarrow_{\circ} y_{2}$. The reactions in a MESSI network satisfy the following rules: if three species are related by $X_{i}+X_{j} \rightarrow_{\circ} X_{k}$ or $X_{k} \rightarrow_{\circ} X_{i}+X_{j}$, then $X_{k}$ is an intermediate species. If two monomolecular complexes consisting of a single core species $X_{i}, X_{j}$ are related by $X_{i} \rightarrow_{\circ} X_{j}$, then there exists $\alpha \geq 1$ such that both belong to $\mathscr{S}^{(\alpha)}$. And if $X_{i}+X_{j} \rightarrow_{\circ} X_{k}+X_{\ell}$ then, there exist $\alpha \neq \beta$ such that $X_{i}, X_{k} \in \mathscr{S}^{(\alpha)}, X_{j}, X_{\ell} \in \mathscr{S}^{(\beta)}$ or $X_{i}, X_{\ell} \in \mathscr{S}^{(\alpha)}, X_{j}, X_{k} \in \mathscr{S}^{(\beta)}$.

A partition in the set of species that satisfies all the previous conditions in the complexes and reactions defines a MESSI structure. There can be many possible partitions that define a MESSI structure of a fixed network. If we have two partitions $\mathscr{S}=\mathscr{S}^{(0)} \sqcup \mathscr{S}^{(1)} \sqcup \mathscr{S}^{(2)} \sqcup$ $\cdots \sqcup \mathscr{S}^{(m)}$ and $\mathscr{S}=\mathscr{S}^{\prime(0)} \sqcup \mathscr{S}^{\prime(1)} \sqcup \mathscr{S}^{\prime(2)} \sqcup \cdots \sqcup \mathscr{S}^{\prime\left(m^{\prime}\right)}$, we say that the first partition refines the second one if and only if $\mathscr{S}^{(0)} \supseteq \mathscr{S}^{(0)}$ and for any $\alpha \geq 1$, there exists $\alpha^{\prime} \geq 1$ such that $\mathscr{S}^{(\alpha)} \subseteq \mathscr{S}^{\prime\left(\alpha^{\prime}\right)}$. This defines a partial order in the set of all possible partitions, and in particular we have the notion of a minimal partition.

Example 5.1. In Section 4 we presented the distributive multisite phosphorylation systems. The following network is an example of a mixed phosphorylation mechanism (partially distributive, partially processive) studied in [26]. The reaction network is as follows:

$$
\begin{array}{ll}
S_{0}+E & \stackrel{k_{1}}{\rightleftarrows} E S_{0} \stackrel{k_{3}}{\rightarrow} S_{1}+E \underset{k_{2}}{\stackrel{k_{4}}{\leftrightarrows}} E S_{1} \stackrel{k_{6}}{\rightarrow} S_{2}+E \\
S_{2}+F & \stackrel{k_{7}}{\rightleftarrows} F S_{2} \stackrel{k_{9}}{\rightarrow} F S_{1} \stackrel{k_{10}}{\rightarrow} S_{0}+F
\end{array}
$$

A MESSI structure of the network is given by the following minimal partition of the species: $\mathscr{S}^{(0)}=\left\{E S_{0}, E S_{1}, F S_{1}, F S_{2}\right\}$ (the intermediate species), $\mathscr{S}^{(1)}=\{E\}, \mathscr{S}^{(2)}=\{F\}$ and $\mathscr{S}^{(3)}=\left\{S_{0}, S_{1}, S_{2}\right\}$. We will use this partition in the next examples featuring this network. Another example of a partition giving a MESSI structure, which is not minimal, is the following: $\mathscr{S}^{\prime(0)}=\left\{E S_{0}, E S_{1}, F S_{1}, F S_{2}\right\}, \mathscr{S}^{\prime(1)}=\{E, F\}$ and $\mathscr{S}^{\prime(2)}=\left\{S_{0}, S_{1}, S_{2}\right\}$.

We now present three digraphs associated to a MESSI network with digraph $G$. First, we introduce the associated digraph $G_{1}$, where the intermediate species are eliminated, that is, with set of species $\mathscr{S}_{1}$. We associated to this set the inherited partition

$$
\mathscr{S}_{1}=\mathscr{S}^{(1)} \bigsqcup \mathscr{S}^{(2)} \bigsqcup \cdots \bigsqcup \mathscr{S}^{(m)} \text {. }
$$

The vertex set of $G_{1}$ consists of all the core complexes. An edge $y \rightarrow y^{\prime}$, with $y, y^{\prime}$ core complexes, belongs to the edge set of $G_{1}$ if and only if $y \rightarrow_{\circ} y^{\prime}$ in $G$. The explicit rate 


$$
\begin{aligned}
S_{0}+E \stackrel{\tau_{1}}{\rightarrow} S_{1}+E \stackrel{\tau_{2}}{\rightarrow} S_{2}+E & \\
& S_{2}+F \stackrel{\tau_{3}}{\rightarrow} S_{0}+F
\end{aligned}
$$

$G_{1}$
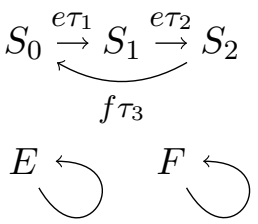

$G_{2}$

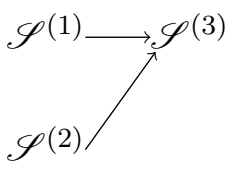

$G_{E}$

Figure 10. The digraphs $G_{1}, G_{2}$ and $G_{E}$ of the network in Example 5.1.

constants of these edges are described in the proof of Theorem 3 in the ESM of [12] (also made explicit in [24]). We will use the following notation for the intermediate species: $\mathscr{S}^{(0)}=\left\{U_{1}, \ldots, U_{p}\right\}$. For each $y \rightarrow \circ y^{\prime}$ in $G$, with $y$ and $y^{\prime}$ core complexes, the reaction constant $\tau(\kappa)$ in $G_{1}$ which gives the label $y \stackrel{\tau(\kappa)}{\longrightarrow} y^{\prime}$, equals:

$$
\tau(\kappa)=\kappa_{y y^{\prime}}+\sum_{k=1}^{p} \kappa_{k} \mu_{k}(\kappa),
$$

where $\kappa_{y y^{\prime}} \geq 0$ is positive when $y \stackrel{\kappa_{y y^{\prime}}}{\longrightarrow} y^{\prime}$ in $G$ (and $\kappa_{y y^{\prime}}=0$ otherwise), and $\kappa_{k} \geq 0$ is positive if $U_{k} \stackrel{\kappa_{k}}{\longrightarrow} y^{\prime}$ and $y \rightarrow_{\circ} U_{k}$ in $G$ (and $\kappa_{k}=0$ otherwise), with $\mu_{k}(\kappa)$ as in (5.8) below.

We next introduce a labeled associated multidigraph $G_{2}$ where we "hide" the concentrations of some of the species in the labels. We keep all monomolecular reactions $X_{i} \rightarrow X_{j}$ in $G_{1}$ and for each reaction $X_{i}+X_{\ell} \stackrel{\tau}{\longrightarrow} X_{j}+X_{m}$ in $G_{1}$, with $X_{i}, X_{j} \in \mathscr{S}^{(\alpha)}, X_{\ell}, X_{m} \in \mathscr{S}^{(\beta)}$, we consider two reactions $X_{i} \stackrel{\tau x_{\ell}}{\longrightarrow} X_{j}$ and $X_{\ell} \stackrel{\tau x_{i}}{\longrightarrow} X_{m}$. In principle this multidigraph $M G_{2}$ might contain loops or parallel edges between any pair of nodes. We obtain the digraph $G_{2}$ by collapsing into one edge all parallel edges of $M G_{2}$. The label of an edge in $G_{2}$ is the sum of the labels of the parallel edges in the multidigraph. By the rules of the reactions in a MESSI network, $G_{2}$ is a linear graph (each node is indicated by a single variable) and the labels on the edges depend on the rate constants but might also depend on the concentrations of some species. We call $G_{2}^{\circ}$ the digraph obtained from the deletion of loops and isolated nodes of $G_{2}$. It can be shown (see Lemma 18 of [24]) that if the partition associated to a MESSI system is minimal, the connected components of the associated digraph $G_{2}$ are in bijection with the subsets $\mathscr{S}^{(\alpha)}$ corresponding to a core species and the set of nodes of the corresponding component equals $\mathscr{S}^{(\alpha)}$.

Finally, given a MESSI system with a minimal partition of the set of species, we define the associated digraph $G_{E}$, whose vertices are the sets $\mathscr{S}^{(\alpha)}$ for $\alpha \geq 1$, and there is an edge from $\mathscr{S}^{(\alpha)}$ to $\mathscr{S}^{(\beta)}$ if there is a species in $\mathscr{S}^{(\alpha)}$ in a label of an edge in $G_{2}^{\circ}$ between species of $\mathscr{S}^{(\beta)}$.

Example 5.2 (Example 5.1, continued). The digraphs $G_{1}, G_{2}$, and $G_{E}$ associated to the network of Example 5.1 are depicted in Figure 10.

In order to give the definition of an $s$-toric MESSI we have to recall some definitions from graph theory. Recall that a vertex in a directed graph has indegree zero if it is not the head of any directed edge and outdegree zero if it is not the tail of any directed edge. A spanning tree of a digraph is a subgraph that contains all the vertices, is connected and acyclic as an undirected graph. An $i$-tree of a graph is a spanning tree where the $i$-th vertex is its unique sink (that is, the only vertex with outdegree zero). Given an $i$-tree $T$, we call $c^{T}$ the product of the labels of all the edges of $T$. An $s$-toric MESSI system is a MESSI system that also satisfies the following conditions: i) for any intermediate complex $y$, there exists a unique core complex $y_{1}$ such that $y_{1} \rightarrow_{\circ} y$, ii) the associated multidigraph $M G_{2}$ does not have parallel edges and the digraph $G_{2}$ is weakly reversible (i.e., for any pair of nodes in the same connected component there is a directed path joining them), iii) for 
each vertex $i$ of $G_{2}^{\circ}$ and any choice of $i$-trees $T, T^{\prime}$ of $G_{2}^{\circ}$, the quotient $c^{T} / c^{T^{\prime}}$ only depends on the rate constants $\tau$. It is interesting to note that even if this definition is restrictive, many of the common enzymatic networks in the literature satisfy these conditions. So, there is a wide applicability of our results.

5.2. Existence of rescalings. The following proposition summarizes some results of [24] and describes the conservation laws as well as the existence of a positive parametrization of the positive steady states. By a positive parametrization of the variety $V$ of positive steady states we mean a $\mathcal{C}^{1}$ and bijective function

$$
\begin{aligned}
\phi: \mathbb{R}_{>0}^{m} & \rightarrow V \cap \mathbb{R}_{>0}^{s}, \\
\bar{x}=\left(\bar{x}_{1}, \ldots, \bar{x}_{m}\right) & \mapsto\left(\phi_{1}(\bar{x}), \ldots, \phi_{n}(\bar{x})\right),
\end{aligned}
$$

for some $m<s$. Proposition 5.3 also features the form of the system when we replace the concentration at steady state of the species by its parametrization into the conservation laws, which is our procedure when we apply our results to the question of determining regions of multistationarity of biochemical reaction networks.

Proposition 5.3. Let $G$ be the underlying digraph of a MESSI system with fixed reaction rate constants $\kappa$. Consider a minimal partition of the set of species as in (5.1) and the associated digraphs $G_{2}$ and $G_{E}$ defined above. Suppose that the system is s-toric, $G_{E}$ has no directed cycles and assume that any pair of nodes in the same connected component of $G_{2}$ are connected by a unique simple path. ${ }^{1}$

Choose $m$ species $X_{i_{1}}, \ldots, X_{i_{m}}$, such that $X_{i_{\alpha}} \in \mathscr{S}^{(\alpha)}$ for $\alpha=1, \ldots, m$. Then, there exists an explicit basis of $m$ conservation laws with coefficients 0,1 and a positive monomial parametrization of the concentrations of the species at steady state in terms of the $m$ concentration variables $x_{i_{1}}, \ldots, x_{i_{m}}$. Moreover, if we replace the concentrations of the species by its parametrization in these conservations laws we obtain a system of the form:

$$
\ell_{\alpha}(x, \kappa):=\sum_{j=1}^{n} \varphi_{\alpha, j}(\kappa) x^{a_{j}}=T_{\alpha}, \quad \alpha=1, \ldots, m,
$$

where $x=\left(x_{i_{1}}, \ldots, x_{i_{m}}\right)$, with $a_{j} \in \mathbb{Z}^{m}-\{0\}$ for each $j=1, \ldots, n$, for some constants $T_{\alpha}$ which are positive if the trajectory intersects the positive orthant for each $\alpha=1, \ldots, m$. Here $\varphi_{\alpha, j}(\kappa)$ is a positive rational function in the reaction rate constants for each $\alpha=$ $1, \ldots, m$ and $j=1, \ldots, n$.

Now, we state the main result of this section, which guarantees that the rescaling of the original parameters $\kappa$ can always be done in our setting. Its proof can be implemented as an algorithm.

Theorem 5.4. Let $G$ be the underlying digraph of a MESSI system. Consider a minimal partition of the set of species, and the associated digraphs $G_{2}$ and $G_{E}$ defined as before. Suppose that the system is s-toric, $G_{E}$ has no directed cycles and assume that any pair of nodes in the same connected component of $G_{2}$ are connected by a unique simple path. Fix $m$ species $X_{i_{1}}, \ldots, X_{i_{m}}$, such that $X_{i_{\alpha}} \in \mathscr{S}^{(\alpha)}$ for $\alpha=1, \ldots, m$ and consider the parametrization and the system (5.4) obtained in Proposition 5.3.

Given $\gamma \in \mathbb{R}_{>0}^{n+1}$, reaction rate constants $\kappa$ and total conservation constants $T_{\alpha}>0$, there exists a choice of positive reaction rate constants $\bar{\kappa}$ such that the positive solutions of the system

$$
\sum_{j=1}^{n} \gamma_{j} \varphi_{\alpha, j}(\kappa) x^{a_{j}}-\gamma_{n+1} T_{\alpha}=0, \quad \alpha=1, \ldots, m
$$

\footnotetext{
${ }^{1} \mathrm{~A}$ simple path is a path that visits each vertex exactly once.
} 
are in bijection with the positive solutions of

$$
\ell_{\alpha}(x, \bar{\kappa})-T_{\alpha}=\sum_{j=1}^{n} \varphi_{\alpha, j}(\bar{\kappa}) x^{a_{j}}-T_{\alpha}=0, \quad \alpha=1, \ldots, m .
$$

Moreover, the reaction rate constants $\bar{\kappa}$ can be obtained from the original constants $\kappa$ by scaling only the rate constants of those reactions coming out from a core complex.

The proofs of these two results are given below. First, we show in the network of Example 5.1 which parameters we rescale in the proof of Theorem 5.4.

Example 5.5 (Example 5.1, continued). It is easy to check that the network of Example 5.1 with the MESSI structure defined before is an s-toric MESSI systems and satisfies the hypotheses of Theorem 5.4. In the proof of this theorem, we show that it is sufficient to rescale the parameters $k_{1}, k_{4}$ and $k_{7}$, which are the rate constants of reactions coming out from the core complexes $S_{0}+E, S_{1}+E$ and $S_{2}+F$ respectively.

We need to introduce the following sets, as in the proof of Theorem 21 of [24].

Definition 5.6. Let $G$ be the underlying digraph of a MESSI system. Consider a minimal partition of the set of species as in (5.1) and the associated digraph $G_{E}$. We define the following subsets of indices:

$$
\begin{aligned}
& L_{0}=\left\{\beta \geq 1 \text { : indegree of } \mathscr{S}^{(\beta)} \text { is } 0\right\} \text {, and for } k \geq 1: \\
& L_{k}=\left\{\beta \geq 1: \text { for any edge } \mathscr{S}^{(\gamma)} \rightarrow \mathscr{S}^{(\beta)} \text { in } G_{E} \text { it holds that } \gamma \in L_{t} \text {, with } t<k\right\} \backslash \bigcup_{t=0}^{k-1} L_{t} .
\end{aligned}
$$

Example 5.7 (Example 5.1, continued). The subsets $L_{k}, k \geq 0$ of Definition 5.6 for the network of Example 5.1 with the MESSI structure defined before are: $L_{0}=\{1,2\}$ and $L_{1}=\{3\}$ (see the corresponding digraph $G_{E}$ at Figure 10).

5.3. The proofs. We will need a series of remarks and technical lemmas in order to prove our main result Theorem 5.4 that ensures that the general method developed in Section 3 can be applied to the determination of regions of multistatinarity for any s-toric MESSI system. We introduce new ideas, but unluckily our results lie heavily on the machinery developed in [24] and they require the reader to consult that paper. We will need some combinatorial definitions that we will recall succintly.

We first give the proof of Proposition 5.3.

Proof of Proposition 5.3. Suppose that we have a minimal partition of the set of species as in (5.1). With our assumptions, the hypotheses of Theorem 12 in [24] are satisfied, then there exist $m$ conservation laws of the form

$$
\ell_{\alpha}(u, x)=T_{\alpha}, \text { where } \ell_{\alpha}(u, x)=\sum_{X_{j} \in \mathscr{S}^{(\alpha)}} x_{j}+\sum_{k \in \operatorname{Int}(\alpha)} u_{k}, \quad \alpha=1, \ldots, m,
$$

for some constants $T_{\alpha}$, which are positive if the trajectory intersects the positive orthant for each $\alpha=1, \ldots, m$, and where $\operatorname{Int}(\alpha) \subset\{1, \ldots, p\}$ is the following set of indices:

$$
\operatorname{Int}(\alpha)=\left\{k: \exists y \rightarrow_{\circ} U_{k} \text {, with } y \text { core complex with one species belonging to } \mathscr{S}^{(\alpha)}\right\} \text {. }
$$

Because the system is an $s$-toric MESSI system, by Proposition 27 of [24], we can obtain the concentration of the intermediate species at steady state in terms of the concentrations of the core species. That proposition states that there are (explicit) rational functions $\mu_{k}(\kappa) \in \mathbb{Q}(\kappa), 1 \leq k \leq p$, such that at steady state:

$$
u_{k}(\boldsymbol{x})=\mu_{k}(\kappa) \boldsymbol{x}^{y}, \quad k=1, \ldots, p,
$$

where here $\boldsymbol{x}$ denotes the vector of variables corresponding to the concentration of core species and $y$ is the unique core complex reacting through intermediates to $U_{k}$ (here we identify the complex $y$ with the corresponding vector in $\mathbb{Z}_{\geq 0}^{s}$ ). Also, as $G_{2}$ is weakly reversible and $G_{E}$ has no direct cycles, we can apply Theorem 21 of [24] to obtain a 
rational parametrization of the concentration of the core species. Consider the subsets $L_{k}, k \geq 0$ as in Definition 5.6. Observe that the set $L_{0}$ is not empty because $G_{E}$ has no direct cycles. Fix $x_{i_{\alpha}} \in \mathscr{S}^{(\alpha)}$ for each $\alpha=1, \ldots, m$. In the proof of Theorem 21 of [24] it is shown that we can then parametrize all the species of $\mathscr{S}^{(\alpha)}$ for $\alpha \in L_{k}$ in terms of $x_{i_{\alpha}}$, species corresponding to core subsets in $L_{t}$ with $t<k$ and the rate constants $\tau$. Under the assumption that any pair of nodes in the same connected component of $G_{2}$ is connected by a single simple path, we can show that this parametrization is a positive monomial parametrization, using Theorem 28 of [24].

Then, the concentration of a core species at steady state can be written as a monomial in terms of the variables $x_{i_{\alpha}}$, for $\alpha=1, \ldots, m$ and the rate constants $\kappa$, and using this and (5.8), the same holds for any intermediate species. We denote by $\left\{a_{1}, \ldots, a_{n}\right\}$ the different monomials that appear in this monomial parametrization. We replace this parametrization in the conservations laws (5.7) and we get a system as in (5.4), where $\varphi_{\alpha, j}(\kappa)$ is the sum of the coefficients in the parametrization of the species that appear in the $\alpha$-th conservation law and have the monomial $x^{a_{j}}$, that is, $\varphi_{\alpha, j}(\kappa)$ is a positive rational function depending on the reaction rate constants $\kappa$.

In order to prove Theorem 5.4 we need some lemmas. The following lemma shows how the values of $\tau(\kappa)$ and $\mu_{k}(\kappa)$ for $k=1, \ldots, p$, depending on the reaction rate constants $\kappa$, are modified if we consider new rate constants $\bar{\kappa}$ obtained from $\kappa$ after scaling by a positive number all constants in a reaction coming out from a core complex.

Lemma 5.8. Let $G$ be the underlying digraph of an s-toric MESSI system, with reaction rate constants $\kappa, \mu_{k}(\kappa)$ as in (5.8). Fix $\ell_{y} \in \mathbb{R}_{>0}$ for each $y$ core complex. Consider the following reaction rate constants $\bar{\kappa}$ obtained from the rate constants $\kappa$ :

$$
\bar{\kappa}_{y y^{\prime}}= \begin{cases}\ell_{y} \kappa_{y y^{\prime}} & \text { if } y \text { is a core complex, } \\ \kappa_{y y^{\prime}} & \text { if } y \text { is not a core complex. }\end{cases}
$$

That is, we multiply the reactions rate constants coming out from a core complex (we multiply by $\ell_{y}$ if the core complex is $y$ ) and we keep fixed the other rate constants (the constants coming out from an intermediate complex). Then, for each $k=1, \ldots, p$ we have

$$
\mu_{k}(\bar{\kappa})=\ell_{y} \mu_{k}(\kappa) \text { if } y \text { is the unique complex core such that } y \rightarrow_{\circ} U_{k} .
$$

Consequently, if $y \stackrel{\tau}{\rightarrow} y^{\prime}$ is in $G_{1}$, then

$$
\tau(\bar{\kappa})=\ell_{y} \tau(\kappa) .
$$

Proof. Following the proofs of Proposition 27 in [24] and Theorem 2 in [12], we recall how to obtain the constants $\mu_{k}(\kappa)$ for fixed reaction rate constants $\kappa$. They build a new linear labeled directed graph $\widehat{G}$ with node set $\mathscr{S}^{(0)} \cup\{*\}$, which consists of collapsing all core complexes into the vertex $*$, and labeled directed edges that are obtained from hiding the core complexes in the labels. For example, $X_{i}+X_{j} \stackrel{\kappa}{\rightarrow} U_{k}$ becomes $* \stackrel{\kappa x_{i} x_{j}}{\longrightarrow} U_{k}$ and $U_{k} \stackrel{\kappa^{\prime}}{\rightarrow} X_{i}+X_{j}$ becomes $U_{k} \stackrel{\kappa^{\prime}}{\rightarrow} *$.

They show, using the Laplacian of a graph and the Matrix-tree Theorem (see $[22,27]$ ), that

for any $k=1, \ldots, p$, where

$$
\mu_{k}(\kappa)=\rho_{k} / \rho,
$$

$$
\rho_{k}=\sum_{T \text { an } U_{k}-\text { tree }} c^{T}, \quad \rho=\sum_{T \text { an } *-\text { tree }} c^{T} .
$$

It is easy to check that every $*$-tree involves labels in $\mathbb{Q}[\kappa]$, and only labels from edges coming out from an intermediate complex. As the system is $s$-toric, for every intermediate complex formed with the intermediate species $U_{k}$, there is a unique core complex $y$ such that $y \rightarrow_{\circ} U_{k}$. Then, every $U_{k}$-tree involves labels in terms of $\kappa$ and the concentrations of the species that form $y$. Moreover, as there must be a path from $*$ to $U_{k}$ in each $U_{k}$-tree, 
then, a label from an edge coming out from $y$ necessarily appears in each tree (and is the unique label from an edge coming out from a core complex). Then, if we consider the constants $\bar{\kappa}$, each label from an edge coming out from $y$ is multiplied by $\ell_{y}$ and then $\mu_{k}(\bar{\kappa})=\ell_{y} \mu_{k}(\kappa)$ if $y \rightarrow_{\circ} U_{k}$, as wanted. The expression of the constants $\tau(\bar{\kappa})$ follows from (5.3).

In the following lemma we give in more detail the form of the positive parametrization given in Proposition 5.3.

Lemma 5.9. With the hypotheses of Theorem 5.4, fix $X_{i_{1}}, \ldots, X_{i_{m}}$ species as in Proposition 5.3, with $X_{i_{\alpha}} \in \mathscr{S}^{(\alpha)}$, for each $\alpha=1, \ldots, m$. Take any other species $X_{i} \in \mathscr{S}^{(\alpha)}$ with $\alpha \in L_{k}, X_{i} \neq X_{i_{\alpha}}$, with $L_{k}$ as in Definition 5.6. Then, the concentration of $X_{i}$ in terms of $x_{i_{1}}, \ldots, x_{i_{m}}$ can be expressed in the form:

$$
x_{i}=\phi(\tau) x_{i_{\alpha}} \underline{x}^{a},
$$

for some $\phi(\tau) \in \mathbb{Q}(\tau)$, where $\underline{x}^{a}$ is a monomial that depends only on variables $x_{i_{\beta}}$ with $\beta \in L_{t}$, with $t<k$. Moreover, $\phi(\tau)$ has the form

$$
\phi(\tau)=\left(\prod_{j=1}^{q} \frac{\tau_{j, 1}}{\tau_{j, 2}}\right) g\left(\tau^{\prime}\right)
$$

for some $q \geq 1$, where $g\left(\tau^{\prime}\right)$ is a rational function of the constants $\tau^{\prime}$, with $\tau^{\prime}$ the label of edges of connected components of $G_{2}$ corresponding to $\mathscr{S}^{(\beta)}$, with $\beta \in L_{t}$, with $t<k$, and $\tau_{j, 1}, \tau_{j, 2}$ label of edges of the connected component of $G_{2}$ corresponding to $\mathscr{S}^{(\alpha)}$, for each $j=1, \ldots, q$.

Proof. We have that any pair of nodes in the component of $G_{2}$ corresponding to $\mathscr{S}^{(\alpha)}$ are connected by a unique simple path. Then, two different (simple) cycles can only share a node in common (if there are two nodes in common, there will be more than a single path connecting one of the nodes to the other, a contradiction). For each species $X_{j} \in \mathscr{S}^{(\alpha)}$, we consider the set of cycles in the subgraph $G_{2}$ that have $X_{j}$ as a node, that is:

$$
\mathcal{C}\left(X_{j}\right)=\left\{C: C \text { is a (simple) cycle with } X_{j} \text { a node of } C\right\} .
$$

Observe that these sets are nonempty because $G_{2}$ is weakly reversible by hypothesis. Now, we define the following subsets of $\mathscr{S}^{(\alpha)}$.

$$
\begin{aligned}
& N_{0}=\left\{X_{i_{\alpha}}\right\}, \\
& N_{q}=\left\{X_{j} \in \mathscr{S}^{(\alpha)}: X_{j} \in C, \text { for some } C \in \mathcal{C}\left(X_{j^{\prime}}\right) \text {, with } X_{j^{\prime}} \in N_{q-1}\right\} \backslash \bigcup_{t=0}^{q-1} N_{t}, q \geq 1 .
\end{aligned}
$$

Suppose that $X_{i} \in N_{q}$, for some $q \geq 1$. By hypothesis, there is a unique simple path between two nodes species in $\mathscr{S}^{(\alpha)}$, so there exist unique species $Z_{0}=X_{i_{\alpha}}, Z_{1}, \ldots, Z_{q}=$ $X_{i}$, such that $Z_{j} \in \mathcal{C}\left(Z_{j-1}\right)$, for $j=1, \ldots, q$. Then, there exist $q$ cycles in $G_{2}$ :

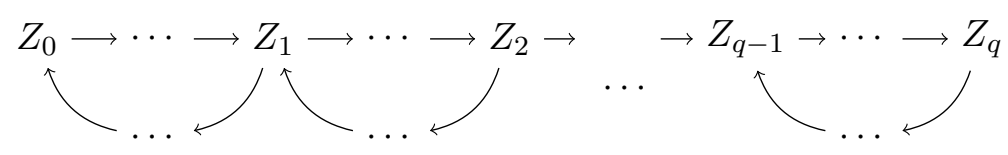

each one of the form:

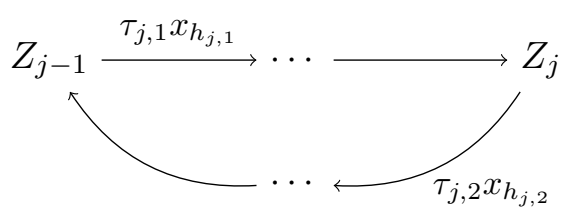


where $x_{h_{j}, 1}, x_{h_{j}, 2}$ are the concentrations of species in core subsets belonging to $L_{t}$ for $t<k$ or are equal to 1. Following the proof of Theorem 28 of [24], we have that at steady state:

$$
\tau_{j, 1} x_{h_{j, 1}} z_{j-1}=\tau_{j, 2} x_{h_{j, 2}} z_{j},
$$

for each $j=1, \ldots, q$. From all these equations, we have that:

$$
x_{i}=z_{q}=\left(\prod_{j=1}^{q} \frac{\tau_{j, 1}}{\tau_{j, 2}}\right)\left(\prod_{j=1}^{q} \frac{x_{h_{j, 1}}}{x_{h_{j, 2}}}\right) x_{i_{\alpha}} .
$$

Using a recursive argument for the variables $x_{h_{j}, 1}, x_{h_{j}, 2}$, we obtain what we wanted.

In the proof of Theorem 5.4 we will show how to modify the rate constants coming out from core complexes. If the digraph $G_{E}$ has no directed cycles, we can consider the sets $L_{k}, k \geq 0$ as in Definition 5.6. Given $k \geq 1$ and $\alpha \in L_{k}$, we denote by $\mathcal{Y}_{\alpha}$ the set of reactant ${ }^{2}$ core complexes which consist only of one species of $\mathscr{S}^{(\alpha)}$ or which consist of one species of $\mathscr{S}^{(\alpha)}$ and one species in a core subset with index in $L_{t}$ with $t<k$.

If $G_{2}$ is weakly reversible, for each $y \in \mathcal{Y}_{\alpha}$, there exist at least one simple cycle $C$ in $G_{2}$ that contains an outgoing edge of the form $X_{i} \stackrel{\tau x_{j}}{\longrightarrow}$ if $y=X_{i}+X_{j}$ or an edge of the form $X_{i} \stackrel{\tau}{\rightarrow}$ if $y=X_{i}$, where $X_{i} \in \mathcal{S}^{(\alpha)}$. In this case, we say that the complex $y$ appears in the simple cycle $C$. We define the following subsets of $\mathcal{Y}_{\alpha}$.

Definition 5.10. Assume $G$ is the underlying digraph of a MESSI system satisfying the hypotheses of Theorem 5.4; in particular, we fix $X_{i_{\alpha}} \in \mathscr{S}^{(\alpha)}$ for each $\alpha=1, \ldots, m$. Let $N_{q}$ and $\mathcal{C}\left(X_{j}\right)$ defined as in the proof of Lemma 5.9. For any $k \geq 1$ and $\alpha \in L_{k}$, we define the following subsets of $\mathcal{Y}_{\alpha}$ :

$$
\begin{aligned}
& M_{0}=\left\{y \in \mathcal{Y}_{\alpha}: \text { one species of } y \text { is } X_{i_{\alpha}}\right\}, \\
& M_{0}^{\prime}=\left\{y \in \mathcal{Y}_{\alpha}: y \text { appears in } C \text { with } C \in \mathcal{C}\left(X_{i_{\alpha}}\right)\right\} \backslash M_{0}, \text { and for } q \geq 1: \\
& M_{q}=\left\{y \in \mathcal{Y}_{\alpha}: \text { one species of } y \text { belongs to } N_{q}\right\} \backslash \bigcup_{t=0}^{q-1}\left(M_{t} \cup M_{t}^{\prime}\right), \\
& \left.M_{q}^{\prime}=\left\{y \in \mathcal{Y}_{\alpha}: y \text { appears in } C \text { with } C \in \mathcal{C}(Z) \text {, for some } Z \in N_{q}\right\}\right) \backslash\left(\bigcup_{t=0}^{q-1}\left(M_{t} \cup M_{t}^{\prime}\right) \cup M_{q}\right) .
\end{aligned}
$$

We clarify in our example the previous definitions.

Example 5.11 (Example 5.1, continued). Consider the network and its MESSI structure of Example 5.1. Choose the species $S_{0} \in \mathscr{S}^{(3)}$. Looking at the connected component corresponding to $\mathscr{S}^{(3)}$ in the digraph $G_{2}$ in Figure 10, the sets $N_{q}$ that appear in the proof of Lemma 5.9 are: $N_{0}=\left\{S_{0}\right\}, N_{1}=\left\{S_{1}, S_{2}\right\}$. The set $\mathcal{C}\left(S_{0}\right)$ consists only of the simple cycle:

$$
S_{0} \underset{f \tau_{3}}{\stackrel{e \tau_{1}}{\longrightarrow} S_{1} \stackrel{e \tau_{2}}{\longrightarrow}} S_{2}
$$

The set $\mathcal{Y}_{3}$ is $\left\{S_{0}+E, S_{1}+E, S_{2}+F\right\}$. The sets $M_{q}$ of Definition 5.10 are: $M_{0}=\left\{S_{0}+E\right\}$, $M_{0}^{\prime}=\left\{S_{1}+E, S_{2}+F\right\}$ (the complexes $S_{1}+E$ and $S_{2}+F$ appear in the the previous cycle of $\left.\mathcal{C}\left(S_{0}\right)\right)$.

Now we are ready to present the proof of Theorem 5.4.

Proof of Theorem 5.4. We can suppose without loss of generality that the coefficient $\gamma_{n+1}$ in the system (5.5) is equal to 1 ; if not, we divide each equation by $\gamma_{n+1}$ and we obtain new values of $\gamma$ for each monomial. Note that $x_{i_{\alpha}}$ is one of the monomials that appears in

\footnotetext{
${ }^{2} \mathrm{~A}$ reactant complex $y$ is a complex for which exists a reaction $y \rightarrow y^{\prime}$.
} 
the system (2.10) for all $\alpha=1, \ldots, m$. We can suppose that the corresponding multiplier $\gamma_{\alpha}$ of $x_{i_{\alpha}}$ in system (2.10) is equal to 1 for all $\alpha$. Otherwise, we change the variables

$$
\gamma_{\alpha} x_{i_{\alpha}}=\bar{x}_{i_{\alpha}} .
$$

In this case, we get a system with new values of the vector $\gamma$, in which the positive solutions are in bijection with the positive solutions of system (5.5).

With these assumptions, we assert that we can transform system (5.5) into system (5.6), just rescaling the rate constants of reactions coming out from a core complex, in a certain order, multiplying each one by an appropriate constant. We consider the sets $L_{k}$, as in Definition 5.6. Recall that $L_{0}$ is no empty because $G_{E}$ has no direct cycles. Because the partition is minimal the subsets of core species $\mathscr{S}^{(\alpha)}$ are in bijection with the connected components of $G_{2}$ and the set of nodes of the corresponding component equals $\mathscr{S}^{(\alpha)}$.

Let $\mathscr{S}^{(\alpha)} \in L_{0}$. We showed in Lemma 5.9 that all the core species in $\mathscr{S}^{(\alpha)}$ can be written in terms of the monomial $x_{i_{\alpha}}$, reaction rate constants and no other variables. If an intermediate complex has its unique core complex reacting to it via intermediates formed with species only in $\mathscr{S}^{(\alpha)}$, then the concentration of the corresponding intermediate species also depends only on $x_{i_{\alpha}}$ and reaction rate constants. That is, all the concentrations of these species have $x_{i_{\alpha}}$ as the corresponding monomial in the parametrization. We supposed that in system (5.5) the monomial $x_{i_{\alpha}}$ is multiplied by $\gamma_{\alpha}=1$, then, there is nothing to rescale.

Now we proceed recursively. Fix $k \geq 1$. Suppose that we have already rescaled properly the reaction rate constants of edges coming out from core complexes whose parametrizations depends only on variables $x_{i_{\beta}}$ with $\beta \in L_{t}$, with $t<k$. Fix one core subset $\mathscr{S}^{(\alpha)}$, with $\alpha \in L_{k}$. We will show how to rescale the rate constants of reactions coming out from complexes in the set $\mathcal{Y}_{\alpha}$, defined above.

The digraph $G_{2}$ is weakly reversible, then we can consider the sets $M_{q}, M_{q}^{\prime}, q \geq 0$, as in Definition 5.10. We are going to rescale the rate constants of reactions coming out from a complex in $M_{0}$, then in $M_{0}^{\prime}$, then in $M_{1}$ and so on, in that order. First, we show how to modify the constants of reactions coming out from a complex in $M_{0}$. Because the system is $s$-toric, each intermediate complex has a unique core complex reacting through intermediates to it. We consider the intermediates complexes such the unique core complex reacting through intermediates to it is in $M_{0}$ or in $M_{0}^{\prime}$ (if there is no one, we don't rescale anything). Suppose then that there is one intermediate complex formed by an intermediate species $U_{\ell}$ such that $y \rightarrow_{\circ} U_{\ell}$, with $y \in M_{0}$ or $y \in M_{0}^{\prime}$. If the core complex $y \in M_{0}$, then $y=X_{i_{\alpha}}$ or $y=X_{i_{\alpha}}+X_{j}$, with $X_{j}$ in a core subset belonging to $L_{t}$, with $t<k$. If $y=X_{i}$, the concentration of $U_{k}$ is $u_{\ell}=\mu_{\ell}(\kappa) x_{i_{\alpha}}$, with $\mu_{\ell}$ as in (5.14), and we are assuming that the monomial $x_{i_{\alpha}}$ is multiplied by $\gamma_{\alpha}=1$. If $y=X_{i_{\alpha}}+X_{j}$, then we can write:

$$
u_{\ell}=\mu_{\ell}(\kappa) x_{i_{\alpha}} x_{j} \text {. }
$$

Now, $x_{j}$ is a concentration of a core species and its parametrization can be written in terms of cores species of subsets in the partition with indices in $L_{t}$, with $t<k$ and reaction rate constants $\tau^{\prime}(\kappa)$, with $\tau^{\prime}(\kappa)$ labels of edges of connected components of $G_{2}$, corresponding to core subsets with indices in $L_{t}$, with $t<k$. We write $x_{j}=g\left(\tau^{\prime}(\kappa)\right) \underline{x}^{a}$, with $\underline{x}^{a}$ a monomial in these other species and $g$ a rational function, and we get:

$$
u_{\ell}=\mu_{\ell}(\kappa) g\left(\tau^{\prime}(\kappa)\right) \underline{x}^{a} x_{i_{\alpha}} .
$$

Suppose that the monomial $\underline{x}^{a} x_{i_{\alpha}}$ appears in system (5.5) multiplied by $\gamma$. Then, we want new reaction rate constants $\bar{\kappa}$ such that:

$$
\gamma \mu_{\ell}(\kappa) g\left(\tau^{\prime}(\kappa)\right)=\mu_{\ell}(\bar{\kappa}) g\left(\tau^{\prime}(\bar{\kappa})\right) .
$$

To ease the notation, we denote $\mu_{\ell}=\mu_{\ell}(\kappa), \tau^{\prime}=\tau^{\prime}(\kappa), \bar{\mu}_{\ell}=\mu_{\ell}(\bar{\kappa}), \bar{\tau}^{\prime}=\tau^{\prime}(\bar{\kappa})$ (and we will denote with a bar the constants depending on $\bar{\kappa}$ and without a bar, the constants depending on $\kappa$ ). The constants $\tau^{\prime}$ have been modified previously by hypothesis (note that a constant $\tau$ can only appear in one edge of $G_{2}$, because of the condition that $G_{E}$ has no cycles) and replaced by the constants $\bar{\tau}^{\prime}$. It is clear that we can do the rescaling: it is 
enough to multiply each reaction constant of reactions coming out from the core complex $y$ by the constant: $\gamma \frac{g\left(\tau^{\prime}\right)}{g\left(\bar{\tau}^{\prime}\right)}$. Then, by Lemma 5.8, we obtain the equality (5.14). Now, if $y \in M_{0}^{\prime}, y$ appears in $C$ with $C \in \mathcal{C}\left(X_{i_{\alpha}}\right)$. Then, $y=X_{i}$ or $y=X_{i}+X_{j^{\prime}}$, with $C$ of the form

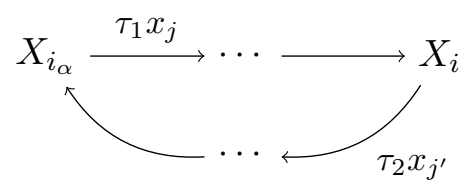

where $x_{j^{\prime}}$ is the concentration of $X_{j^{\prime}}$ or is equal to 1 (if $y=X_{i}$ ), and similarly for $x_{j}$. Then, we have at steady state:

$$
u_{\ell}=\mu_{\ell} x_{i} x_{j^{\prime}}=\mu_{\ell} \frac{\tau_{1}}{\tau_{2}} x_{i_{\alpha}} x_{j}
$$

That is, $u_{\ell}$ depends on the concentrations of the species of the complex $X_{i_{\alpha}}+X_{j}$, which belongs to $M_{0}$. We then modify the reaction rate constants coming out of $X_{i_{\alpha}}+X_{j}$ multiplying it by an appropiate constant in a similar way as we did in the previous case, looking at the value of $\gamma$ that appears in the corresponding monomial (note that if we modified these constants before, the previous rescaling also works for this case). Note that when later we modify the constants of the complex $y=X_{i}+X_{j^{\prime}}$ which belongs to $M_{0}^{\prime}$ (we will see how to do this), the rescaling will be coherent. That is, if we multiply the constants of each reaction coming out from $X_{i_{\alpha}}+X_{j}$ by $\nu_{1}$, and the constants coming out from $y$ by $\nu_{2}$ the rescaling will be coherent if we have:

$$
\bar{\mu}_{\ell} \frac{\bar{\tau}_{1}}{\bar{\tau}_{2}}=\nu_{1} \mu_{\ell} \frac{\tau_{1}}{\tau_{2}}
$$

but this holds by Lemma 5.8:

$$
\bar{\mu}_{\ell} \frac{\bar{\tau}_{1}}{\bar{\tau}_{2}}=\nu_{2} \mu_{\ell} \frac{\nu_{1} \tau_{1}}{\nu_{2} \tau_{2}}=\nu_{1} \mu_{\ell} \frac{\tau_{1}}{\tau_{2}},
$$

where $\bar{\mu}_{\ell}, \bar{\tau}_{1}, \bar{\tau}_{2}$ denotes the values of the functions $\mu_{\ell}, \tau_{1}, \tau_{2}$ corresponding to the new constants $\bar{\kappa}$. We modify all the reactions rate constants coming out of complexes $y$ belonging to $M_{0}$ in this way: looking at intermediates complexes $U_{\ell}$ such that $y \rightarrow_{\circ} U_{\ell}$ or $y^{\prime} \rightarrow_{\circ} U_{\ell}$, with $y^{\prime} \in M_{0}^{\prime}$ and such that in the parametrization of the intermediate species appears the monomial corresponding to the complex $y$. If there is no such intermediate complex we multiply the constants by 1 . Also, we observe that with this rescaling, we modified all the constants $\tau$ that label an edge in $G_{2}$ of the form $X_{i_{\alpha}} \stackrel{\tau x_{j}}{\longrightarrow}$.

Now, we show how to rescale the constants of complexes in $M_{0}^{\prime}$. Let $y \in M_{0}^{\prime}$, then $y=X_{i}$ or $y=X_{i}+X_{j^{\prime}}$ and we have a cycle as we showed previously in this proof. By Lemma 5.9, the concentration $x_{i}$ is of the form

$$
x_{i}=\frac{\tau_{1}}{\tau_{2}} g\left(\tau^{\prime}\right) \underline{x}^{a} x_{i_{\alpha}},
$$

where $\tau^{\prime}$ are labels of edges of connected components of $G_{2}$, corresponding to core subsets belong to $L_{t}$, with $t<k, g(\tau)$ a rational form and $\underline{x}^{a}$ a monomial in variables in core subsets belong to $L_{t}$, with $t<k$. The constant $\tau_{1}$ and the constants $\tau^{\prime}$ have been already modified by the constants $\overline{\tau_{1}}$ and $\bar{\tau}^{\prime}$ respectively. It is clear that we can do the rescaling if we modify $\tau_{2}$. If $\gamma$ is the constant that multiplies the monomial $\underline{x}^{a} x_{i_{\alpha}}$ in system (5.5), we want

$$
\gamma \frac{\tau_{1}}{\tau_{2}} g\left(\tau^{\prime}\right)=\frac{\overline{\tau_{1}}}{\bar{\tau}_{2}} g\left(\bar{\tau}^{\prime}\right)
$$

and we get this equality if we multiply each rate constant of a reaction coming out from $y$ by the constant $\frac{\overline{\tau_{1}} g\left(\overline{\tau^{\prime}}\right)}{\gamma \tau_{1} g\left(\tau^{\prime}\right)}$ and we apply Lemma 5.8. We do this for all complexes in $M_{0}^{\prime}$.

We proceed recursively rescaling the remaining constants of reactions coming out from complexes in $M_{q}$, and then from $M_{q}^{\prime}$, for each $q$. We first modify the constants of complexes 


$$
\begin{aligned}
& S_{0}+E \stackrel{\tau_{0}}{\rightarrow} S_{1}+E \stackrel{\tau_{1}}{\rightarrow} S_{2}+E \\
& S_{2}+F \stackrel{\nu_{1}}{\rightarrow} S_{1}+F \stackrel{\nu_{0}}{\rightarrow} S_{0}+F
\end{aligned}
$$

$G_{1}$

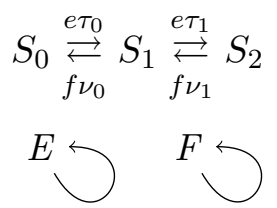

$G_{2}$

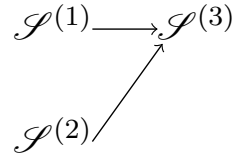

$G_{E}$

Figure 11 . The digraphs $G_{1}, G_{2}$ and $G_{E}$ of the double phosphorylation.

in $M_{q}$, by looking at the parametrization of intermediate species as we did when we showed how to rescale the constants of reactions coming out from complexes in $M_{0}$. After that, we modify the constants of complexes in $M_{q}^{\prime}$ by looking the concentration of the core species in $\mathscr{S}^{(\alpha)}$ that appear in the complex, as we did for the complexes in $M_{0}^{\prime}$. Then, we can rescale all the complexes of $\mathcal{Y}_{\alpha}$, for all $\alpha \in L_{k}$. We can proceed for all $k, k \geq 1$, in order, and then we are done.

Example 5.12. The distributive multisite phosphorylation systems showed in Section 4 are all in the hypotheses of Theorem 5.4. A MESSI structure of the network for the double phosporylation $(n=2)$ is given by this minimal partition of the species:

$\mathscr{S}^{(0)}=\left\{E S_{0}, E S_{1}, F S_{1}, F S_{2}\right\}$ (the intermediate species), $\mathscr{S}^{(1)}=\{E\}, \mathscr{S}^{(2)}=\{F\}$ and $\mathscr{S}^{(3)}=\left\{S_{0}, S_{1}, S_{2}\right\}$.

The digraphs $G_{1}, G_{2}$ and $G_{E}$ are depicted in Figure 11. It is easy to check the conditions of Theorem 5.4 in this case. Following the proof of this theorem, we can show which parameters are sufficient to rescale. For this case is sufficient to modify $k_{\mathrm{on}_{0}}, k_{\mathrm{on}_{1}}, \ell_{\mathrm{on}_{0}}$ and $\ell_{\mathrm{on}_{1}}$, the rate constants of reactions coming out of core complexes.

\section{ACKNOWLEDGMENT}

The authors are grateful to the Kurt and Alice Wallenberg Foundation and to the Institut Mittag-Leffler, Sweden, for their support to start and to make progress on this work. Our thanks go also to the Mathematics Department of the Royal Institute of Technology, Sweden, for the wonderful hospitality we enjoyed. AD and MG are partially supported by UBACYT 20020100100242, CONICET PIP 11220150100473, and ANPCyT PICT 2013-1110, Argentina.

\section{REFERENCES}

[1] M. Banaji, C. Pantea. The inheritance of nondegenerate multistationarity in chemical reaction networks. SIAM J. Appl. Math. 78(2) (2018), 1105-1130.

[2] F. Bihan, F. Santos, P-J. Spaenlehauer. A polyhedral method for sparse systems with many positive solutions. Preprint, available at: arXiv:1804.05683 (2018).

[3] C. Conradi, E. Feliu, M. Mincheva, C. Wiuf. Identifying parameter regions for multistationarity. PLoS computational biology, 13(10), (2017), e1005751.

[4] C. Conradi, D. Flockerzi, J. Raisch. Multistationarity in the activation of a MAPK: Parametrizing the relevant region in parameter space. Mathematical biosciences 211 (1) (2012), 105-131.

[5] C. Conradi, M. Mincheva. Catalytic constants enable the emergence of bistability in dual phosphorylation. J. R. Soc. Interface (2014), rsif20110664.

[6] C. Conradi, A. Shiu. A global convergence result for processive multisite phosphorylation systems. Bull. Math. Biol. 77 (1) (2015), 126-155.

[7] G. Craciun, M. Feinberg. Multiple equilibria in complex chemical reaction networks: I. The injectivity property. SIAM J. Appl. Math. 65 (2005), 1526-1546.

[8] G. Craciun, M. Feinberg. Multiple equilibria in complex chemical reaction networks: II. The Species-Reactions Graph. SIAM J. Appl. Math. 66(4) (2006), 1321-1338.

[9] J. de Loera, J.A. Rambau, F. Santos. Triangulations: Structures for Algorithms and Applications, vol. 25. Springer-Verlag, 2010.

[10] A. Dickenstein. Biochemical reaction networks: an invitation for algebraic geometers. MCA 2013, Contemporary Mathematics 656 (2016), 65-83. 
[11] E. Feliu, C. Wiuf. Enzyme-sharing as a cause of multi-stationarity in signaling systems J. R. Soc. Interface 9 (2012), 1224-1232.

[12] E. Feliu, C. Wiuf Simplifying biochemical models with intermediate species. J. R. Soc. Interface (2013) 10: 20130484.

[13] D. Flockerzi, C. Conradi. Subnetwork analysis for multistationarity in mass action kinetics. J. of Physics: Conference Series 138 (2008), 012006.

[14] K. Gatermann, M. Wolfrum. Bernstein's second theorem and Viro's method for sparse polynomial systems in chemistry. Advances in Applied Mathematics, 34(2), (2005), 252-294

[15] E. Gawrilow, M. Joswig. polymake: a framework for analyzing convex polytopes. Polytopes, combinatorics and computation (Oberwolfach, 1997), 43-73, DMV Sem., 29, Birkhäuser, Basel, (2000).

[16] I. M. Gelfand, M. M. Kapranov, A. V. Zelevinsky. Discriminants, resultants, and multidimensional determinants. Mathematics: Theory \& Applications. Birkhauser Boston Inc., Boston, MA, 1994.

[17] M. Giaroli, F. Bihan, A. Dickenstein. Regions of multistationarity in cascades of GoldbeterKoshland loops, preprint (2018), to be posted soon in arXiv.org.

[18] A. Goldbeter, D. E. Koshland. An amplified sensitivity arising from covalent modification in biological systems. Proceedings of the National Academy of Sciences, 78(11), (1981) 6840-6844.

[19] K. Holstein, D. Flockerzi, C. Conradi. Multistationarity in Sequential Distributed Multisite Phosphorylation Networks. Bull. Math. Biol. 75 (11) (2013), 2028-2058.

[20] B. Joshi, A. Shiu. Atoms of multistationarity in chemical reaction networks. J. Math. Chem. 51(1) (2013), 153-178.

[21] V. B. Kothamachu, E. Feliu, L. Cardelli, O. S. Soyer. Unlimited multistability and Boolean logic in microbial signaling. J. R. Soc. Interface (2015), 1220150234 .

[22] I. Mirzaev, J. Gunawardena. Laplacian dynamics on general graphs. Bull. Math. Biol., 75 (2013), 2118-2149.

[23] S. Mueller, E. Feliu, G. Regensburger, C. Conradi, A. Shiu, A. Dickenstein. Sign conditions for injectivity of generalized polynomial maps with applications to chemical reaction networks and real algebraic geometry. FoCM Journal 16 (1) (2016), 69-97.

[24] M. Pérez Millán, A. Dickenstein. The structure of MESSI biochemical networks. To appear: SIAM J. Appl. Dyn. Syst. (2018), available at: arXiv:1612.08763.

[25] A. M. Stock, V. L. Robinson, P. N. Goudreau. Two-component signal transduction. Annual review of biochemistry, 69(1) (2000), 183-215.

[26] T. Suwanmajo, J. Krishnan. Mixed mechanisms of multi-site phosphorylation. Journal of the Royal Society Interface (2015), 1220141405.

[27] W. T. Tutte, The dissection of equilateral triangles into equilateral triangles. Proc. Cambridge Philos. Soc., 44 (1948), 463-482.

[28] L. Wang, E. Sontag. On the number of steady states in a multiple futile cycle. J. Math. Biol. 57(1) (2008), 29-52.

Laboratoire de Mathématiques, Université Savoie Mont Blanc, 73376 Le Bourget-Du-LaC Cedex, France

E-mail address: Frederic.Bihan@univ-savoie.fr

URL: http://www. lama.univ-savoie.fr/〜 bihan/

Dto. de Matemática, FCEn, Universidad de Buenos Aires, and imas (UBA-COniCET), Ciudad Universitaria, Pab. I, C1428EGA Buenos Aires, Argentina

E-mail address: alidick@dm.uba.ar

URL: http://mate.dm.uba.ar/〜alidick

Dto. de Matemática, FCEn, Universidad de Buenos Aires, and imas (UBA-COniCET), Ciudad Universitaria, Pab. I, C1428EGA Buenos Aires, Argentina

E-mail address: mgiaroli@dm.uba.ar 